\title{
The Szegö Kernel on a Sewn Riemann Surface
}

\author{
Michael P. Tuite and Alexander Zuevsky * \\ School of Mathematics, Statistics and Applied Mathematics, \\ National University of Ireland, Galway, Ireland
}

September 4, 2018

\begin{abstract}
We describe the Szegö kernel on a higher genus Riemann surface in terms of Szegö kernel data coming from lower genus surfaces via two explicit sewing procedures where either two Riemann surfaces are sewn together or a handle is sewn to a Riemann surface. We consider in detail the examples of the Szegö kernel on a genus two Riemann surface formed by either sewing together two punctured tori or by sewing a twice-punctured torus to itself. We also consider the modular properties of the Szegö kernel in these cases.
\end{abstract}

\section{Introduction}

The purpose of this paper is to provide an explicit description of the Szegö kernel [Sz], [HS], [Sc], [F1] on a higher genus Riemann surface in terms of Szegö kernel data coming from lower genus surfaces. We exploit two explicit sewing procedures where either two lower genus Riemann surfaces are sewn together or else a handle is sewn to a lower genus Riemann surface. We also consider in some detail the construction and modular properties of the Szegö kernel on a genus two Riemann surface formed either by sewing two tori together or by sewing a handle on to a torus. This paper is a further development of the theory of partition and $n$-point correlation functions on Riemann surfaces for vertex operator algebras (e.g. [FLM], [Ka]) as described

\footnotetext{
*Supported by a Science Foundation Ireland Research Frontiers Programme Grant
} 
in [T], [MT1, [MT1, [MT2, [MT3], [MT4, [MTZ]. Our main motivation is to lay the foundations for the explicit construction of the partition and $n$-point correlation functions for a fermionic vertex operator super algebra on higher genus Riemann surfaces [TZ1, ,TZ2]. (The central role played by the Szegö kernel for such systems has been long appreciated in theoretical physics [RS], [R], [DVFHLS], DVPFHLS]). Our ultimate aim is to develop a fully rigorous theory of higher genus partition and $n$-point functions along the lines of Zhu's theory at genus one [Z]. Thus, to name but a few applications, one might eventually study Siegel (sub)group modular invariance, the Freidan-Shenker conjecture concerning the reconstructability of a conformal field theory from the genus $g$ partition function at all genera [FS], higher genus bosonization etc. However, this present paper may also be of interest to readers outside the vertex operator algebra community.

We begin in Section 2 with a review of some basic aspects of the theory of Riemann surfaces [FK], [Sp, [F1],[F2],[Mu]. We then define and discuss properties of the Szegö kernel, which is a meromorphic $\left(\frac{1}{2}, \frac{1}{2}\right)$ differential with a simple pole structure and prescribed multiplicities on the cycles of the Riemann surface [Sz], [HS], [Sc], [F1].

In Section 3 we describe the Szegö kernel on a genus $g_{1}+g_{2}$ Riemann surface $\Sigma^{\left(g_{1}+g_{2}\right)}$ obtained by sewing two lower genus Riemann surfaces $\Sigma^{\left(g_{1}\right)}$ and $\Sigma^{\left(g_{2}\right)}$. This is similar to the approach of refs. [Y] and [MT1, for computing the period matrix and other related structures on $\Sigma^{\left(g_{1}+g_{2}\right)}$ in terms of lower genus data. Following [MT1, we refer to this sewing scheme as the $\epsilon$-formalism where $\epsilon$ is a complex sewing parameter which forms part of the data according to which the sewing is performed (see Figure 1 below). In particular, we introduce an infinite block matrix

$$
Q=\left(\begin{array}{cc}
0 & \xi F_{1} \\
-\xi F_{2} & 0
\end{array}\right),
$$

where $F_{1}$ and $F_{2}$ are infinite matrices whose entries are certain weighted moments of the Szegö kernels on $\Sigma^{\left(g_{1}\right)}$ and $\Sigma^{\left(g_{2}\right)}$, respectively, and $\xi \in\{ \pm \sqrt{-1}\}$. The matrix $I-Q$, where $I$ is the infinite identity matrix, plays a crucial role here (and in the sequel [TZ1]). In particular, we show that $I-Q$ is invertible for small enough $\epsilon$. $(I-Q)^{-1}$ then forms part of the expression of the genus $g_{1}+g_{2}$ Szegö kernel in terms of the lower genus Szegö kernel data as proved in Theorem 3.6. In Theorem 3.9 we further show that the determinant $\operatorname{det}(I-Q)$ is well-defined and is a non-vanishing holomorphic function 
for small enough $\epsilon$. Finally, we describe the example of the Szegö kernel on a genus two Riemann surface formed by sewing two tori and verify its modular transformation properties under the modular group which preserves the sewing scheme. This example is extensively exploited in [TZ1].

Section 4 is devoted to development of the corresponding formalism in the case that $\Sigma^{(g+1)}$ of genus $g+1$ is obtained by self-sewing a handle to a genus

$g$ Riemann surface $\Sigma^{(g)}$ with complex sewing parameter $\rho$. We refer to this as the $\rho$-formalism. This case is more technical due to the extra multiplicities on the two new cycles associated with the sewing handle. This leads us to introduce an analogue of (1), namely, an infinite matrix $T$ whose entries are determined by weighted moments of certain genus $g$ objects related to the Szegö kernel on $\Sigma^{(g)}$ and the new multiplicities. We show that $I-T$ is invertible for suitably small $\rho$ and in Theorem 4.6 express the Szegö kernel on $\Sigma^{(g+1)}$ in terms of $(I-T)^{-1}$ and other genus $g$ Szegö kernel data. In Theorem 4.7 we show that the determinant $\operatorname{det}(I-T)$ is well-defined and holomorphic for suitably small $\rho$. We conclude with two examples of sewing a handle to a Riemann sphere to obtain a torus and sewing a handle to a torus to obtain genus two Riemann surface. The modular transformation properties of the genus two Szegö kernel are also verified under the modular group preserving this $\rho$-sewing scheme. This example will be extensively exploited in [TZ2].

\section{The Szegö Kernel on a Riemann Surface}

Consider a compact Riemann surface $\Sigma$ of genus $g$ with canonical homology cycle basis $a_{1}, \ldots, a_{g}, b_{1}, \ldots, b_{g}$. In general there exists $g$ holomorphic 1 -forms $\nu_{i}, i=1, \ldots, g$ which we may normalize by (e.g. [FK], $[\mathrm{Sp}$ )

$$
\oint_{a_{i}} \nu_{j}=2 \pi i \delta_{i j} .
$$

The genus $g$ period matrix $\Omega$ is defined by

$$
\Omega_{i j}=\frac{1}{2 \pi i} \oint_{b_{i}} \nu_{j}
$$

for $i, j=1, \ldots, g$. $\Omega$ is symmetric with positive imaginary part i.e. $\Omega \in \mathbb{H}_{g}$, the Siegel upper half plane. The canonical intersection form on cycles is 
preserved under the action of the symplectic group $S p(2 g, \mathbb{Z})$ where

$$
\left(\begin{array}{l}
b \\
a
\end{array}\right) \rightarrow\left(\begin{array}{c}
\tilde{b} \\
\tilde{a}
\end{array}\right)=\left(\begin{array}{cc}
A & B \\
C & D
\end{array}\right)\left(\begin{array}{l}
b \\
a
\end{array}\right), \quad\left(\begin{array}{cc}
A & B \\
C & D
\end{array}\right) \in S p(2 g, \mathbb{Z}) .
$$

This induces the modular action on $\mathbb{H}_{g}$

$$
\Omega \rightarrow \tilde{\Omega}=(A \Omega+B)(C \Omega+D)^{-1} .
$$

It is useful to introduce the normalized differential of the second kind defined by $[\mathrm{Sp}]$, [Mu], [F1]:

$$
\omega(x, y) \sim \frac{d x d y}{(x-y)^{2}} \quad \text { for } x \sim y
$$

for local coordinates $x, y$, with normalization $\int_{a_{i}} \omega(x, \cdot)=0$ for $i=1, \ldots, g$. Using the Riemann bilinear relations, one finds that $\nu_{i}(x)=\oint_{b_{i}} \omega(x, \cdot)$.

We also introduce the normalized differential of the third kind

$$
\omega_{p_{2}-p_{1}}(x)=\int_{p_{1}}^{p_{2}} \omega(x, \cdot)
$$

for which $\oint_{a_{i}} \omega_{p_{2}-p_{1}}=0$ and $\omega_{p_{2}-p_{1}}(x) \sim \frac{(-1)^{a}}{x-p_{a}} d x$ for $x \sim p_{a}$ and $a=1,2$.

We recall the definition of the theta function with real characteristics e.g. [Mu, [F1, FK]

$$
\vartheta\left[\begin{array}{l}
\alpha \\
\beta
\end{array}\right](z \mid \Omega)=\sum_{m \in \mathbb{Z}^{g}} \exp (i \pi(m+\alpha) . \Omega .(m+\alpha)+(m+\alpha) \cdot(z+2 \pi i \beta)),
$$

for $\alpha=\left(\alpha_{i}\right), \beta=\left(\beta_{i}\right) \in \mathbb{R}^{g}, z=\left(z_{i}\right) \in \mathbb{C}^{g}$ and $i=1, \ldots, g$ with

$$
\begin{aligned}
\vartheta\left[\begin{array}{c}
\alpha \\
\beta
\end{array}\right](z+2 \pi i(\Omega . r+s) \mid \Omega) & =e^{2 \pi i \alpha \cdot s} e^{-2 \pi i \beta . r} e^{-i \pi r \cdot \Omega \cdot r-r . z} \vartheta\left[\begin{array}{l}
\alpha \\
\beta
\end{array}\right](z \mid \Omega), \\
\vartheta\left[\begin{array}{c}
\alpha+r \\
\beta+s
\end{array}\right](z \mid \Omega) & =e^{2 \pi i \alpha \cdot s} \vartheta\left[\begin{array}{c}
\alpha \\
\beta
\end{array}\right](z \mid \Omega),
\end{aligned}
$$

for $r, s \in \mathbb{Z}^{g}$.

There exists a (nonsingular and odd) character $\left[\begin{array}{l}\gamma \\ \delta\end{array}\right]$ such that $[\mathrm{Mu}],[\mathrm{F} 1]$

$$
\vartheta\left[\begin{array}{l}
\gamma \\
\delta
\end{array}\right](0 \mid \Omega)=0, \quad \partial_{z_{i}} \vartheta\left[\begin{array}{l}
\gamma \\
\delta
\end{array}\right](0 \mid \Omega) \neq 0
$$


Let

$$
\zeta(x)=\sum_{i=1}^{g} \partial_{z_{i}} \vartheta\left[\begin{array}{l}
\gamma \\
\delta
\end{array}\right](0 \mid \Omega) \nu_{i}(x)
$$

a holomorphic 1-form, and let $\zeta(x)^{\frac{1}{2}}$ denote the form of weight $\frac{1}{2}$ on the double cover $\widetilde{\Sigma}$ of $\Sigma$. We also refer to $\zeta(x)^{\frac{1}{2}}$ as a (double-valued) $\frac{1}{2}$-form on $\Sigma$. We define the prime form $E(x, y)$ by

$$
E(x, y)=\frac{\vartheta\left[\begin{array}{l}
\gamma \\
\delta
\end{array}\right]\left(\int_{y}^{x} \nu \mid \Omega\right)}{\zeta(x)^{\frac{1}{2}} \zeta(y)^{\frac{1}{2}}} \sim(x-y) d x^{-\frac{1}{2}} d y^{-\frac{1}{2}} \quad \text { for } x \sim y,
$$

where $\int_{y}^{x} \nu=\left(\int_{y}^{x} \nu_{i}\right) \in \mathbb{C}^{g} . E(x, y)=-E(y, x)$ is a holomorphic differential form of weight $\left(-\frac{1}{2},-\frac{1}{2}\right)$ on $\widetilde{\Sigma} \times \widetilde{\Sigma}$. $E(x, y)$ has multipliers along the $a_{i}$ and $b_{j}$ cycles in $x$ given by 1 and $e^{-i \pi \Omega_{j j}-\int_{y}^{x} \nu_{j}}$ respectively [F1].

The normalized differentials of the second and third kind can be expressed in terms of the prime form $[\mathrm{Mu}]$

$$
\begin{aligned}
\omega(x, y) & =\partial_{x} \partial_{y} \log E(x, y) d x d y \\
\omega_{p-q}(x) & =\partial_{x} \log \frac{E(x, p)}{E(x, q)} d x .
\end{aligned}
$$

Conversely, we can also express the prime form in terms of $\omega$ by [F2]

$$
E(x, y)=\lim _{p \rightarrow x, q \rightarrow y}\left[\sqrt{(x-p)(q-y)} \exp \left(-\frac{1}{2} \int_{y}^{x} \omega_{p-q}\right)\right] d x^{-\frac{1}{2}} d y^{-\frac{1}{2}} .
$$

We define the Szegö kernel [Sc], [HS], [F1] for $\vartheta\left[\begin{array}{l}\alpha \\ \beta\end{array}\right](0 \mid \Omega) \neq 0$ as follows

$$
S\left[\begin{array}{l}
\theta \\
\phi
\end{array}\right](x, y \mid \Omega)=\frac{\vartheta\left[\begin{array}{l}
\alpha \\
\beta
\end{array}\right]\left(\int_{y}^{x} \nu \mid \Omega\right)}{\vartheta\left[\begin{array}{l}
\alpha \\
\beta
\end{array}\right](0 \mid \Omega) E(x, y)},
$$

where $\theta=\left(\theta_{i}\right), \phi=\left(\phi_{i}\right) \in U(1)^{n}$ for

$$
\theta_{j}=-e^{-2 \pi i \beta_{j}}, \quad \phi_{j}=-e^{2 \pi i \alpha_{j}}, \quad j=1, \ldots, g .
$$

\footnotetext{
${ }^{1}$ Note that our definition differs from that of refs. $\mathrm{Mu}, \mathrm{F} 1$ by a factor of -1 .
} 
It follows from (9) that (16) is a function of $e^{2 \pi i \alpha_{i}}$ and $e^{2 \pi i \beta_{i}}$. The further factors of -1 in (17) are included for later convenience. The Szegö kernel has multipliers along the $a_{i}$ and $b_{j}$ cycles in $x$ given by $-\phi_{i}$ and $-\theta_{j}$ respectively and is a meromorphic $\left(\frac{1}{2}, \frac{1}{2}\right)$-form on $\widetilde{\Sigma} \times \widetilde{\Sigma}$ satisfying:

$$
\begin{aligned}
& S\left[\begin{array}{l}
\theta \\
\phi
\end{array}\right](x, y) \sim \frac{1}{x-y} d x^{\frac{1}{2}} d y^{\frac{1}{2}} \quad \text { for } x \sim y, \\
& S\left[\begin{array}{l}
\theta \\
\phi
\end{array}\right](x, y)=-S\left[\begin{array}{l}
\theta^{-1} \\
\phi^{-1}
\end{array}\right](y, x),
\end{aligned}
$$

where $\theta^{-1}=\left(\theta_{i}^{-1}\right)$ and $\phi^{-1}=\left(\phi_{i}^{-1}\right)$. Note that the skew-symmetry property (19) implies $S\left[\begin{array}{l}\theta \\ \phi\end{array}\right](x, y)$ has multipliers along the $a_{i}$ and $b_{j}$ cycles in $y$ given by $-\phi_{i}^{-1}$ and $-\theta_{j}^{-1}$ respectively.

Finally, we describe the modular invariance of the Szegö kernel under the symplectic group $S p(2 g, \mathbb{Z})$ where we find [F1]

$$
S\left[\begin{array}{c}
\tilde{\theta} \\
\tilde{\phi}
\end{array}\right](x, y \mid \tilde{\Omega})=S\left[\begin{array}{l}
\theta \\
\phi
\end{array}\right](x, y \mid \Omega),
$$

with $\tilde{\Omega}$ of (5) and where $\tilde{\theta}_{j}=-e^{-2 \pi i \tilde{\beta}_{j}}, \tilde{\phi}_{j}=-e^{2 \pi i \tilde{\alpha}_{j}}$ for

$$
\left(\begin{array}{c}
-\tilde{\beta} \\
\tilde{\alpha}
\end{array}\right)=\left(\begin{array}{cc}
A & B \\
C & D
\end{array}\right)\left(\begin{array}{c}
-\beta \\
\alpha
\end{array}\right)+\frac{1}{2}\left(\begin{array}{c}
-\operatorname{diag}\left(A B^{T}\right) \\
\operatorname{diag}\left(C D^{T}\right)
\end{array}\right),
$$

where $\operatorname{diag}(M)$ denotes the diagonal elements of a matrix $M$.

For a Riemann surface of genus one described by an oriented torus $\mathbb{C} / \Lambda$ for lattice $\Lambda=2 \pi i(\mathbb{Z} \tau \oplus \mathbb{Z})$ for $\tau \in \mathbb{H}_{1}$, the genus one prime form is $E^{(1)}(x, y)=$ $K(x-y, \tau) d x^{-\frac{1}{2}} d y^{-\frac{1}{2}}$ where

$$
K(z, \tau)=\frac{\vartheta_{1}(z, \tau)}{\partial_{z} \vartheta_{1}(0, \tau)}
$$

for $z \in \mathbb{C}$ and $\tau \in \mathbb{H}_{1}$ and where $\vartheta_{1}(z, \tau)=\vartheta\left[\begin{array}{c}\frac{1}{2} \\ \frac{1}{2}\end{array}\right](z, \tau)$.

For $(\theta, \phi) \neq(1,1)$ with $\theta=-e^{-2 \pi i \beta}$ and $\phi=-e^{2 \pi i \alpha}$ the genus one Szegö kernel is

$$
S^{(1)}\left[\begin{array}{l}
\theta \\
\phi
\end{array}\right](x, y \mid \tau)=P_{1}\left[\begin{array}{l}
\theta \\
\phi
\end{array}\right](x-y, \tau) d x^{\frac{1}{2}} d y^{\frac{1}{2}},
$$


where

$$
\begin{aligned}
P_{1}\left[\begin{array}{l}
\theta \\
\phi
\end{array}\right](z, \tau) & =\frac{\vartheta\left[\begin{array}{l}
\alpha \\
\beta
\end{array}\right](z, \tau)}{\vartheta\left[\begin{array}{l}
\alpha \\
\beta
\end{array}\right](0, \tau)} \frac{1}{K(z, \tau)}, \\
& =-\sum_{k \in \mathbb{Z}} \frac{q_{z}^{k+\lambda}}{1-\theta^{-1} q^{k+\lambda}}
\end{aligned}
$$

is a 'twisted' Weierstrass function [MTZ] for $q_{z}=e^{z}$ and with $\phi=\exp (2 \pi i \lambda)$ for $0 \leq \lambda<1$. The genus one modular group $S L(2, \mathbb{Z})$ acts in this case with (20) and (21) following from

$$
P_{1}\left(\gamma\left[\begin{array}{l}
\theta \\
\phi
\end{array}\right]\right)(\gamma z \mid \gamma \tau)=(c \tau+d) P_{1}\left[\begin{array}{l}
\theta \\
\phi
\end{array}\right](z \mid \tau)
$$

with

$$
\gamma \tau=\frac{a \tau+b}{c \tau+d}, \quad \gamma z=\frac{z}{c \tau+d}
$$

for $\gamma=\left(\begin{array}{ll}a & b \\ c & d\end{array}\right) \in S L(2, \mathbb{Z})$ and

$$
\gamma\left[\begin{array}{l}
\theta \\
\phi
\end{array}\right]=\left[\begin{array}{l}
\theta^{a} \phi^{b} \\
\theta^{c} \phi^{d}
\end{array}\right]
$$

We also have a Laurant expansion [MTZ]

$$
P_{1}\left[\begin{array}{l}
\theta \\
\phi
\end{array}\right](z, \tau)=\frac{1}{z}-\sum_{n \geq 1} E_{n}\left[\begin{array}{l}
\theta \\
\phi
\end{array}\right](\tau) z^{n-1},
$$

for twisted Eisenstein series defined by

$$
\begin{aligned}
E_{n}\left[\begin{array}{l}
\theta \\
\phi
\end{array}\right](\tau)= & -\frac{B_{n}(\lambda)}{n !}+\frac{1}{(n-1) !} \sum_{r \geq 0} \frac{(r+\lambda)^{n-1} \theta^{-1} q^{r+\lambda}}{1-\theta^{-1} q^{r+\lambda}} \\
& +\frac{(-1)^{n}}{(n-1) !} \sum_{r \geq 1} \frac{(r-\lambda)^{n-1} \theta q^{r-\lambda}}{1-\theta q^{r-\lambda}},
\end{aligned}
$$

for $n \geq 1$ and where $B_{n}(\lambda)$ is the Bernoulli polynomial defined by

$$
\frac{q_{z}^{\lambda}}{q_{z}-1}=\frac{1}{z}+\sum_{n \geq 1} \frac{B_{n}(\lambda)}{n !} z^{n-1} .
$$

For $(\theta, \phi)=(1,1)$ and $n \geq 2$ the twisted Eisenstein series reduce to the standard elliptic Eisenstein series with $E_{n}(\tau)=0$ for $n$ odd. 


\section{The Szegö Kernel on Two Sewn Riemann Surfaces}

\subsection{The $\epsilon$-Formalism Sewing Scheme}

We review the Yamada [Y] formalism for 'sewing' together two Riemann surfaces $\Sigma^{\left(g_{a}\right)}$ of genus $g_{a}$ for $a=1,2$ to form a surface of genus $g_{1}+g_{2}$. Following [MT1, we refer to this sewing scheme as the $\epsilon$-formalism.

Choose a local coordinate $z_{a}$ on $\Sigma^{\left(g_{a}\right)}$ in the neighborhood of a point $p_{a}$, and consider the closed disk $\left|z_{a}\right| \leq r_{a}$ for $r_{a}>0$, sufficiently small. Let $\epsilon$ be a complex sewing parameter with $|\epsilon| \leq r_{1} r_{2}$ and excise the disk

$$
\left\{z_{a}:\left|z_{a}\right| \leq|\epsilon| / r_{\bar{a}}\right\} \subset \Sigma^{\left(g_{a}\right)},
$$

to form a punctured surface

$$
\widehat{\Sigma}^{\left(g_{a}\right)}=\Sigma^{\left(g_{a}\right)} \backslash\left\{z_{a}:\left|z_{a}\right| \leq|\epsilon| / r_{\bar{a}}\right\} .
$$

Here and below, we use the convention

$$
\overline{1}=2, \quad \overline{2}=1 .
$$

Define the annulus $\mathcal{A}_{a}=\left\{z_{a}:|\epsilon| / r_{\bar{a}} \leq\left|z_{a}\right| \leq r_{a}\right\} \subset \widehat{\Sigma}^{\left(g_{a}\right)}$ and identify $\mathcal{A}_{1}$ and $\mathcal{A}_{2}$ as a single region $\mathcal{A}=\mathcal{A}_{1} \simeq \mathcal{A}_{2}$ via the sewing relation

$$
z_{1} z_{2}=\epsilon
$$

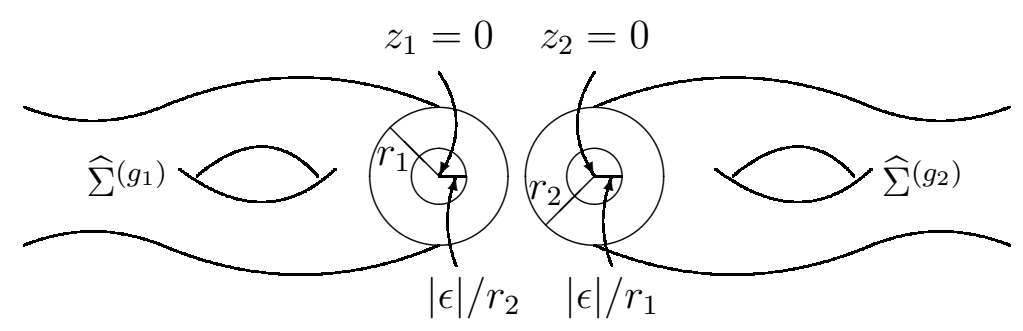

Fig. 1: Sewing Two Riemann Surfaces 
In this way we obtain a compact Riemann surface $\Sigma^{\left(g_{1}+g_{2}\right)}=\left\{\widehat{\Sigma}^{g_{1}} \backslash \mathcal{A}_{1}\right\} \cup$ $\left\{\widehat{\Sigma}^{\left(g_{2}\right)} \backslash \mathcal{A}_{2}\right\} \cup \mathcal{A}$ of genus $g_{1}+g_{2}$. By construction, $\Sigma^{\left(g_{1}+g_{2}\right)}$ degenerates into $\Sigma^{\left(g_{1}\right)}$ and $\Sigma^{\left(g_{2}\right)}$ in the limit $\epsilon \rightarrow 0$.

The form $\omega^{\left(g_{1}+g_{2}\right)}$ on $\Sigma^{\left(g_{1}+g_{2}\right)}$ can be found in terms of data coming from $\omega^{\left(g_{a}\right)}$ on $\widehat{\Sigma}^{\left(g_{a}\right)}[\mathrm{Y}] . \Sigma^{\left(g_{1}+g_{2}\right)}$ inherits a homology cycle basis labeled $\left\{a_{s_{1}}, b_{s_{1}}\right.$ : $\left.s_{1}=1, \ldots, g_{1}\right\}$ and $\left\{a_{s_{2}}, b_{s_{2}}: s_{2}=g_{1}+1, \ldots, g_{1}+g_{2}\right\}$ from $\Sigma^{\left(g_{1}\right)}$ and $\Sigma^{\left(g_{2}\right)}$ respectively. This allows us to compute the normalized 1-forms $\nu_{i}^{\left(g_{1}+g_{2}\right)}$ and the period matrix $\Omega_{i j}^{\left(g_{1}+g_{2}\right)}$. In particular, we find [Y], [MT1]

Theorem $3.1 \omega^{\left(g_{1}+g_{2}\right)}, \nu_{i}^{\left(g_{1}+g_{2}\right)}$ and $\Omega_{i j}^{\left(g_{1}+g_{2}\right)}$ are holomorphic in $\epsilon$ for $|\epsilon|<$ $r_{1} r_{2}$ with

$$
\begin{aligned}
& \omega^{\left(g_{1}+g_{2}\right)}(x, y)=\delta_{a b} \omega^{\left(g_{a}\right)}(x, y)+O(\epsilon), \\
& \nu_{s_{b}}^{\left(g_{1}+g_{2}\right)}(x)=\delta_{a b} \nu_{s_{a}}^{\left(g_{a}\right)}(x)+O(\epsilon), \\
& \Omega_{s_{a} t_{b}}^{\left(g_{1}+g_{2}\right)}=\delta_{a b} \Omega_{s_{a} t_{a}}^{\left(g_{a}\right)}+O(\epsilon),
\end{aligned}
$$

for $x \in \widehat{\Sigma}^{\left(g_{a}\right)}, y \in \widehat{\Sigma}^{\left(g_{b}\right)}$ and $a, b=1,2$ and where $s_{a}$, $t_{b}$ label the inherited homology basis.

The explicit form of $\omega^{\left(g_{1}+g_{2}\right)}, \nu_{i}^{\left(g_{1}+g_{2}\right)}$ and $\Omega_{i j}^{\left(g_{1}+g_{2}\right)}$ is described in [Y], [MT1].

\subsection{The Szegö Kernel in the $\epsilon$-Formalism}

We now determine the Szegö kernel on the Riemann surface $\Sigma^{\left(g_{1}+g_{2}\right)}$ in terms of data coming from Szegö kernel

$$
S^{\left(g_{a}\right)}(x, y)=S^{\left(g_{a}\right)}\left[\begin{array}{l}
\theta^{\left(g_{a}\right)} \\
\phi^{\left(g_{a}\right)}
\end{array}\right](x, y),
$$

on the surface $\Sigma^{\left(g_{a}\right)}$ for $a=1,2$. We adopt the abbreviated notation of the left hand side of (31) when there is no ambiguity. Similarly, the Szegö kernel on $\Sigma^{\left(g_{1}+g_{2}\right)}$ is denoted by

$$
S^{\left(g_{1}+g_{2}\right)}(x, y)=S^{\left(g_{1}+g_{2}\right)}\left[\begin{array}{l}
\theta^{\left(g_{1}+g_{2}\right)} \\
\phi^{\left(g_{1}+g_{2}\right)}
\end{array}\right](x, y),
$$

with periodicities $\left(\theta_{s_{a}}^{\left(g_{1}+g_{2}\right)}, \phi_{s_{a}}^{\left(g_{1}+g_{2}\right)}\right)=\left(\theta_{s_{a}}^{\left(g_{a}\right)}, \phi_{s_{a}}^{\left(g_{a}\right)}\right)$ on the inherited homology basis 2

\footnotetext{
${ }^{2}$ Note that we exclude those Riemann theta characteristics for which (32) exists but where either of the lower genus theta functions vanishes i.e. we assume that (31) exists for $a=1,2$.
} 
We next describe $S^{\left(g_{1}+g_{2}\right)}(x, y)$ in terms of $S^{\left(g_{a}\right)}(x, y)$. We first show that

Theorem $3.2 S^{\left(g_{1}+g_{2}\right)}$ is holomorphic in $\epsilon^{\frac{1}{2}}$ for $|\epsilon|<r_{1} r_{2}$ with

$$
S^{\left(g_{1}+g_{2}\right)}(x, y)=\left\{\begin{array}{l}
S^{\left(g_{a}\right)}(x, y)+O(\epsilon), \quad \text { for } x, y \in \widehat{\Sigma}^{\left(g_{a}\right)}, \\
O\left(\epsilon^{\frac{1}{2}}\right), \quad \text { for } x \in \widehat{\Sigma}^{\left(g_{a}\right)}, y \in \widehat{\Sigma}^{\left(g_{\bar{a}}\right)}
\end{array}\right.
$$

Proof. Applying Theorem 3.1 to (8) we have

$$
\begin{aligned}
\vartheta\left[\begin{array}{l}
\alpha^{\left(g_{1}+g_{2}\right)} \\
\beta^{\left(g_{1}+g_{2}\right)}
\end{array}\right]\left(z^{\left(g_{1}+g_{2}\right)} \mid \Omega^{\left(g_{1}+g_{2}\right)}\right)= & \vartheta\left[\begin{array}{l}
\alpha^{\left(g_{1}\right)} \\
\beta^{\left(g_{1}\right)}
\end{array}\right]\left(z^{\left(g_{1}\right)} \mid \Omega^{\left(g_{1}\right)}\right) \vartheta\left[\begin{array}{l}
\alpha^{\left(g_{2}\right)} \\
\beta^{\left(g_{2}\right)}
\end{array}\right]\left(z^{\left(g_{2}\right)} \mid \Omega^{\left(g_{2}\right)}\right) \\
& +O(\epsilon)
\end{aligned}
$$

with $\left(\alpha^{\left(g_{1}+g_{2}\right)}\right)=\left(\alpha_{1}^{\left(g_{1}\right)}, \ldots, \alpha_{g_{1}}^{\left(g_{1}\right)}, \alpha_{1}^{\left(g_{2}\right)}, \ldots, \alpha_{g_{2}}^{\left(g_{2}\right)}\right)$ etc. We firstly show that the genus $g_{1}+g_{2}$ prime form obeys

$$
E^{\left(g_{1}+g_{2}\right)}(x, y)=\left\{\begin{array}{l}
E^{\left(g_{a}\right)}(x, y)+O(\epsilon), \quad \text { for } x, y \in \widehat{\Sigma}^{\left(g_{a}\right)} \\
O\left(\epsilon^{-\frac{1}{2}}\right), \quad \text { for } x \in \widehat{\Sigma}^{\left(g_{a}\right)}, y \in \widehat{\Sigma}^{\left(g_{\bar{a}}\right)}
\end{array}\right.
$$

For the genus $g_{1}+g_{2}$ odd characteristic of (10) we find from (33) that either $\vartheta\left[\begin{array}{l}\gamma^{\left(g_{1}\right)} \\ \delta^{\left(g_{1}\right)}\end{array}\right](0) \neq 0$ or $\vartheta\left[\begin{array}{l}\gamma^{\left(g_{2}\right)} \\ \delta^{\left(g_{2}\right)}\end{array}\right](0) \neq 0$ on the lower genus surfaces. Hence it follows that $\zeta^{\left(g_{1}+g_{2}\right)}(x) \zeta^{\left(g_{1}+g_{2}\right)}(y)=O(\epsilon)$ for $x \in \widehat{\Sigma}^{\left(g_{a}\right)}, y \in \widehat{\Sigma}^{\left(g_{\bar{a}}\right)}$ for the 1 -form (11). We also note that

$$
\int_{y}^{x} \nu_{s_{b}}^{\left(g_{1}+g_{2}\right)}=\left\{\begin{array}{l}
\int_{y}^{x} \nu_{s_{a}}^{\left(g_{a}\right)}+O(\epsilon), \quad \text { for } x, y \in \widehat{\Sigma}^{\left(g_{a}\right)}, \\
\delta_{a b} \int_{p_{a}}^{x} \nu_{s_{a}}^{\left(g_{a}\right)}+\delta_{\bar{a} b} \int_{y}^{p_{\bar{a}}} \nu_{s_{\bar{a}}}^{\left(g_{\bar{a}}\right)}+O(\epsilon),
\end{array} \text { for } x \in \widehat{\Sigma}^{\left(g_{a}\right)}, y \in \widehat{\Sigma}^{\left(g_{\bar{a}}\right)},\right.
$$

from which it follows that $E^{\left(g_{1}+g_{2}\right)}(x, y)=O\left(\epsilon^{-\frac{1}{2}}\right)$ for $x \in \widehat{\Sigma}^{\left(g_{a}\right)}$ and $y \in \widehat{\Sigma}^{\left(g_{\bar{a}}\right)}$.

We next determine $E^{\left(g_{1}+g_{2}\right)}(x, y)$ for $x, y \in \widehat{\Sigma}^{\left(g_{a}\right)}$. The differential $\omega^{\left(g_{1}+g_{2}\right)}$ for $x, y \in \widehat{\Sigma}^{\left(g_{a}\right)}$ obeys

$$
\omega^{\left(g_{1}+g_{2}\right)}(x, y)-\omega^{\left(g_{a}\right)}(x, y)=a_{a}(x) X_{\bar{a} \bar{a}} a_{a}^{T}(y)=O(\epsilon),
$$

where $a_{a}(x) X_{\bar{a} \bar{a}} a_{a}^{T}(y)=\sum_{k, l \geq 1} a_{a}(x, k) X_{\bar{a} \bar{a}}(k, l) a_{a}(y, l)$ with $a_{a}(x, k)$ a certain 1-form on $\widehat{\Sigma}^{\left(g_{a}\right)}$ and $X_{\bar{a} \bar{a}}(k, l)$ an infinite matrix determined from genus $g_{1}$ and $g_{2}$ data (see [MT1] for details). It follows from (15) that

$$
E^{\left(g_{1}+g_{2}\right)}(x, y)=E^{\left(g_{a}\right)}(x, y) e^{-\frac{1}{2} b_{a} X_{\bar{a} \bar{a}} b_{a}^{T}}=E^{\left(g_{a}\right)}(x, y)+O(\epsilon),
$$


where $b_{a}(k)=\int_{y}^{x} a_{a}(\cdot, k)$. Thus (34) holds. We then apply Theorem 3.1. (33), (34) and (35) to (16) to prove the result.

We next remark that for $x, z_{a} \in \widehat{\Sigma}^{\left(g_{a}\right)}$ then $S^{\left(g_{a}\right)}\left(x, z_{a}\right) S^{\left(g_{1}+g_{2}\right)}\left(z_{a}, y\right)$ is a meromorphic 1-form (cf. [HS]) in $z_{a}$ periodic on the $\Sigma^{\left(g_{a}\right)}$ cycles (cf. (19])) with simple poles described by (18) where

$$
\begin{aligned}
S^{\left(g_{a}\right)}\left(x, z_{a}\right) S^{\left(g_{1}+g_{2}\right)}\left(z_{a}, y\right) & \sim \frac{d z_{a}}{x-z_{a}} S^{\left(g_{1}+g_{2}\right)}(x, y) \text { for } z_{a} \sim x \\
S^{\left(g_{a}\right)}\left(x, z_{a}\right) S^{\left(g_{1}+g_{2}\right)}\left(z_{a}, y\right) & \sim \frac{d z_{a}}{z_{a}-y} S^{\left(g_{a}\right)}(x, y) \text { for } z_{a} \sim y \text { if } y \in \widehat{\Sigma}^{\left(g_{a}\right)} .
\end{aligned}
$$

A similar behavior holds for $S^{\left(g_{1}+g_{2}\right)}\left(x, z_{b}\right) S^{\left(g_{b}\right)}\left(z_{b}, y\right)$ as a meromorphic 1form in $z_{b}$. This allows us to determine the following integral equations

Proposition 3.3 The Szegö kernel on $\Sigma^{\left(g_{1}+g_{2}\right)}$ is given by

$$
\begin{aligned}
S^{\left(g_{1}+g_{2}\right)}(x, y) & =\delta_{a b} S^{\left(g_{a}\right)}(x, y)-\frac{1}{2 \pi i} \oint_{\mathcal{C}_{a}\left(z_{a}\right)} S^{\left(g_{a}\right)}\left(x, z_{a}\right) S^{\left(g_{1}+g_{2}\right)}\left(z_{a}, y\right),(38) \\
& =\delta_{a b} S^{\left(g_{a}\right)}(x, y)+\frac{1}{2 \pi i} \oint_{\mathcal{C}_{b}\left(z_{b}\right)} S^{\left(g_{1}+g_{2}\right)}\left(x, z_{b}\right) S^{\left(g_{b}\right)}\left(z_{b}, y\right),
\end{aligned}
$$

for $x \in \widehat{\Sigma}^{\left(g_{a}\right)}, y \in \widehat{\Sigma}^{\left(g_{b}\right)}$ for $a, b=1,2$ and where $\mathcal{C}_{a}\left(z_{a}\right) \subset \mathcal{A}_{a}$ denotes $a$ closed anti-clockwise oriented contour parameterized by $z_{a}$ surrounding the puncture at $z_{a}=0$ on $\widehat{\Sigma}^{\left(g_{a}\right)}$.

Proof. Let $\sigma_{a}$ be a contour on $\widehat{\Sigma}^{\left(g_{a}\right)}$ surrounding $\mathcal{A}_{a}$ and the given points $x$ (and $y$, if $a=b$ ) on $\widehat{\Sigma}^{\left(g_{a}\right)}$ (see Fig. 2) 3

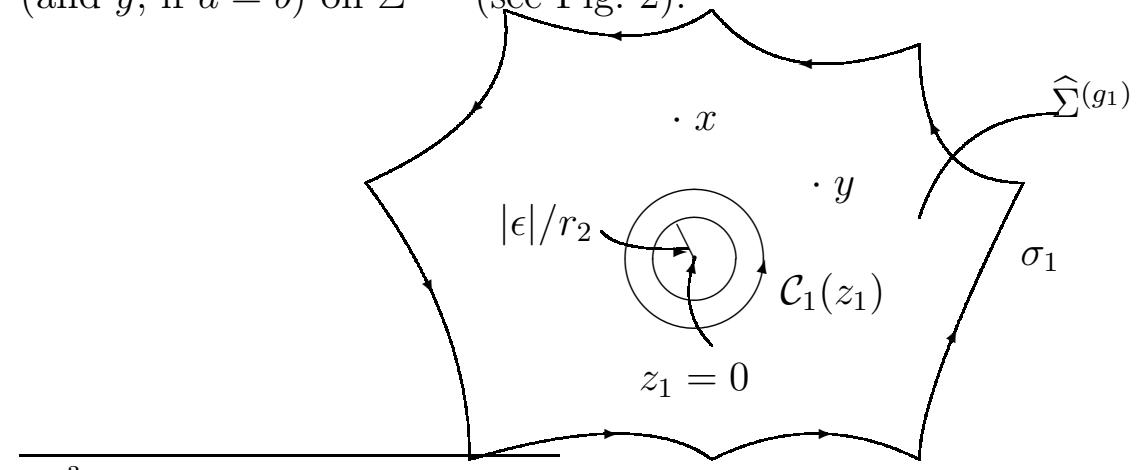

\footnotetext{
${ }^{3} \sigma_{a}$ may be construed as being the boundary of the simple-connected covering space for $\Sigma^{\left(g_{a}\right)}$ as illustrated in Fig. 2 for a genus two surface.
} 
Fig. 2: Example with $x, y \in \widehat{\Sigma}^{\left(g_{1}\right)}$

From Cauchy's theorem $\oint_{\sigma_{a}} S^{\left(g_{a}\right)}\left(x, z_{a}\right) S^{\left(g_{1}+g_{2}\right)}\left(z_{a}, y\right)=0$ and hence (37) gives

$0=-S^{\left(g_{1}+g_{2}\right)}(x, y)+\delta_{a b} S^{\left(g_{a}\right)}(x, y)+\frac{1}{2 \pi i} \oint_{\mathcal{C}_{a}\left(z_{a}\right)} S^{\left(g_{a}\right)}\left(x, z_{a}\right) S^{\left(g_{1}+g_{2}\right)}\left(z_{a}, y\right)$,

giving (38). Considering $S^{\left(g_{1}+g_{2}\right)}\left(x, z_{b}\right) S^{\left(g_{b}\right)}\left(z_{b}, y\right)$ leads to (39).

Similarly to [MT1] we define weighted moments for $S^{\left(g_{1}+g_{2}\right)}$ by

$$
\begin{aligned}
X_{a b}(k, l, \epsilon) & =X_{a b}\left[\begin{array}{l}
\theta^{\left(g_{1}+g_{2}\right)} \\
\phi^{\left(g_{1}+g_{2}\right)}
\end{array}\right](k, l, \epsilon) \\
& =\frac{\epsilon^{\frac{1}{2}(k+l-1)}}{(2 \pi i)^{2}} \oint_{\mathcal{C}_{a}(x)} \oint_{\mathcal{C}_{b}(y)} x^{-k} y^{-l} S^{\left(g_{1}+g_{2}\right)}(x, y) d x^{\frac{1}{2}} d y^{\frac{1}{2}},
\end{aligned}
$$

for $k, l \geq 1$. From (19) it follows that

$$
X_{a b}\left[\begin{array}{l}
\theta^{\left(g_{1}+g_{2}\right)} \\
\phi^{\left(g_{1}+g_{2}\right)}
\end{array}\right](k, l, \epsilon)=-X_{b a}\left[\begin{array}{c}
\left(\theta^{\left(g_{1}+g_{2}\right)}\right)^{-1} \\
\left(\phi^{\left.\left(g_{1}+g_{2}\right)^{-1}\right)}\right.
\end{array}\right](l, k, \epsilon) .
$$

We denote by $X_{a b}=\left(X_{a b}(k, l, \epsilon)\right)$ the infinite matrix indexed by $k, l \geq 1$.

We also define various moments for $S^{\left(g_{a}\right)}(x, y)$. These provide the data used to construct $S^{\left(g_{1}+g_{2}\right)}(x, y)$. Define holomorphic $\frac{1}{2}$-forms on $\widehat{\Sigma}^{\left(g_{a}\right)}$ by

$$
\begin{aligned}
& h_{a}(k, x, \epsilon)=\quad h_{a}\left[\begin{array}{l}
\theta^{\left(g_{a}\right)} \\
\phi^{\left(g_{a}\right)}
\end{array}\right](k, x, \epsilon)=\frac{\epsilon^{\frac{k}{2}-\frac{1}{4}}}{2 \pi i} \oint_{\mathcal{C}_{a}\left(z_{a}\right)} S^{\left(g_{a}\right)}\left(x, z_{a}\right) z_{a}^{-k} d z_{a}^{\frac{1}{2}}, \\
& \bar{h}_{a}(k, y, \epsilon)=\bar{h}_{a}\left[\begin{array}{l}
\theta^{\left(g_{a}\right)} \\
\phi^{\left(g_{a}\right)}
\end{array}\right](k, y, \epsilon)=\frac{\epsilon^{\frac{k}{2}-\frac{1}{4}}}{2 \pi i} \oint_{\mathcal{C}_{a}\left(z_{a}\right)} S^{\left(g_{a}\right)}\left(z_{a}, y\right) z_{a}^{-k} d z_{a}^{\frac{1}{2}},
\end{aligned}
$$

and introduce infinite row vectors $h_{a}(x)=\left(h_{a}(k, x)\right), \bar{h}_{a}(x)=\left(\bar{h}_{a}(k, x)\right)$ indexed by $k \geq 1$. From (19) it follows that

$$
\bar{h}_{a}\left[\begin{array}{l}
\theta^{\left(g_{a}\right)} \\
\phi^{\left(g_{a}\right)}
\end{array}\right](k, x, \epsilon)=-h_{a}\left[\begin{array}{c}
\left(\theta^{\left(g_{a}\right)}\right)^{-1} \\
\left(\phi^{\left(g_{a}\right)}\right)^{-1}
\end{array}\right](k, x, \epsilon) .
$$


Finally, we define the moment matrix

$$
\begin{aligned}
F_{a}(k, l, \epsilon) & =F_{a}\left[\begin{array}{l}
\theta^{\left(g_{a}\right)} \\
\phi^{\left(g_{a}\right)}
\end{array}\right](k, l, \epsilon) \\
& =\frac{\epsilon^{\frac{1}{2}(k+l-1)}}{(2 \pi i)^{2}} \oint_{\mathcal{C}_{a}(x) \mathcal{C}_{a}(y)} \oint_{x^{-k} y^{-l} S^{\left(g_{a}\right)}(x, y) d x^{\frac{1}{2}} d y^{\frac{1}{2}}} \\
& =\frac{\epsilon^{\frac{k}{2}-\frac{1}{4}}}{2 \pi i} \oint_{\mathcal{C}_{a}(x)} x^{-k} h_{a}(l, x) d x^{\frac{1}{2}}=\frac{\epsilon^{\frac{l}{2}-\frac{1}{4}}}{2 \pi i} \oint_{\mathcal{C}_{a}(y)} y^{-l} \bar{h}_{a}(k, y) d y^{\frac{1}{2}}
\end{aligned}
$$

$F_{a}(k, l, \epsilon)$ obeys a skew-symmetry property from (19) similar to (41). We may invert (42)-(45) using (18) to find for $x, y \in \widehat{\Sigma}^{\left(g_{a}\right)}$ that

$$
\begin{aligned}
S^{\left(g_{a}\right)}(x, y) & =\left[\frac{1}{x-y}+\sum_{k, l \geq 1} \epsilon^{-\frac{1}{2}(k+l-1)} F_{a}(k, l, \epsilon) x^{k-1} y^{l-1}\right] d x^{\frac{1}{2}} d y^{\frac{1}{2}} \\
& =\sum_{k \geq 1} \epsilon^{-\frac{k}{2}+\frac{1}{4}} h_{a}(k, x) y^{k-1} d y^{\frac{1}{2}} \\
& =\sum_{l \geq 1} \epsilon^{-\frac{l}{2}+\frac{1}{4}} x^{l-1} \bar{h}_{a}(l, y) d x^{\frac{1}{2}}
\end{aligned}
$$

We are now in a position to express $S^{\left(g_{1}+g_{2}\right)}(x, y)$ in terms of the lower genus data. From the sewing relation (30) we have $d z_{a}=-\epsilon \frac{d z_{\bar{a}}}{z_{\bar{a}}^{2}}$ so that

$$
d z_{a}^{\frac{1}{2}}=(-1)^{\bar{a}} \xi \epsilon^{\frac{1}{2}} \frac{d z_{\bar{a}}^{\frac{1}{2}}}{z_{\bar{a}}}
$$

where $\xi \in\{ \pm \sqrt{-1}\}$ determines the square root branch chosen. We then find

Proposition $3.4 S^{\left(g_{1}+g_{2}\right)}(x, y)$ is given by

$$
S^{\left(g_{1}+g_{2}\right)}(x, y)= \begin{cases}S^{\left(g_{a}\right)}(x, y)+h_{a}(x) X_{\bar{a} \bar{a}} \bar{h}_{a}^{T}(y), & \text { for } x, y \in \widehat{\Sigma}^{\left(g_{a}\right)}, \\ h_{a}(x)\left(\xi(-1)^{\bar{a}} I-X_{\bar{a} a}\right) \bar{h}_{\bar{a}}^{T}(y), & \text { for } x \in \widehat{\Sigma}^{\left(g_{a}\right)}, y \in \widehat{\Sigma}^{\left(g_{\bar{a}}\right)}\end{cases}
$$

where $I$ denotes the infinite identity matrix and $T$ the transpose. 
Proof. Consider $x, y \in \widehat{\Sigma}^{\left(g_{1}\right)}$. Noting that $\mathcal{C}_{1}\left(z_{1}\right)$ may be deformed to $-\mathcal{C}_{2}\left(z_{2}\right)$ on $\mathcal{A}$ via (30) we find

$$
\begin{aligned}
& S^{\left(g_{1}+g_{2}\right)}(x, y)-S^{\left(g_{1}\right)}(x, y)=-\frac{1}{2 \pi i} \oint_{\mathcal{C}_{1}\left(z_{1}\right)} S^{\left(g_{1}\right)}\left(x, z_{1}\right) S^{\left(g_{1}+g_{2}\right)}\left(z_{1}, y\right) \\
= & -\sum_{k \geq 1} h_{1}(k, x) \frac{\epsilon^{-\frac{k}{2}+\frac{1}{4}}}{2 \pi i} \oint_{\mathcal{C}_{1}\left(z_{1}\right)} S^{\left(g_{1}+g_{2}\right)}\left(z_{1}, y\right) z_{1}^{k-1} d z_{1}^{\frac{1}{2}} \\
= & \xi \sum_{k \geq 1} h_{1}(k, x) \frac{\epsilon^{\frac{k}{2}-\frac{1}{4}}}{2 \pi i} \oint_{\mathcal{C}_{2}\left(z_{2}\right)} S^{\left(g_{1}+g_{2}\right)}\left(z_{2}, y\right) z_{2}^{-k} d z_{2}^{\frac{1}{2}} \\
= & \xi \sum_{k \geq 1} h_{1}(k, x) \frac{\epsilon^{\frac{k}{2}-\frac{1}{4}}}{(2 \pi i)^{2}} \oint_{\mathcal{C}_{2}\left(z_{2}\right)} \oint_{\mathcal{C}_{1}\left(u_{1}\right)} S^{\left(g_{1}+g_{2}\right)}\left(z_{2}, u_{1}\right) S^{\left(g_{1}\right)}\left(u_{1}, y\right) z_{2}^{-k} d z_{2}^{\frac{1}{2}} \\
= & -\xi^{2} \sum_{k, l \geq 1} h_{1}(k, x) \bar{h}_{1}(l, y) \frac{\epsilon^{\frac{1}{2}(k+l-1)}}{(2 \pi i)^{2}} \oint_{\mathcal{C}_{2}\left(z_{2}\right)} \oint_{\mathcal{C}_{2}\left(u_{2}\right)} S^{\left(g_{1}+g_{2}\right)}\left(z_{2}, u_{2}\right) z_{2}^{-k} u_{2}^{-l} d z_{2}^{\frac{1}{2}} d u_{2}^{\frac{1}{2}} \\
= & h_{1}(x) X_{22} \bar{h}_{1}^{T}(y),
\end{aligned}
$$

using (38), (47), (49), (48), (39) and (49) again, respectively. Thus we recover the first line of (50) for $a=b=1$. A similar analysis holds for $a=b=2$. 
For $x \in \widehat{\Sigma}^{\left(g_{1}\right)}, y \in \widehat{\Sigma}^{\left(g_{2}\right)}$ we find that

$$
\begin{aligned}
& S^{\left(g_{1}+g_{2}\right)}(x, y)=-\frac{1}{2 \pi i} \oint_{\mathcal{C}_{1}\left(z_{1}\right)} S^{\left(g_{1}\right)}\left(x, z_{1}\right) S^{\left(g_{1}+g_{2}\right)}\left(z_{1}, y\right) \\
= & \xi \sum_{k \geq 1} \frac{\epsilon^{\frac{k}{2}-\frac{1}{4}}}{2 \pi i} \oint_{\mathcal{C}_{2}\left(z_{2}\right)} z_{2}^{-k} d z_{2}^{\frac{1}{2}} h_{1}(k, x) \\
& \cdot\left(S^{\left(g_{2}\right)}\left(z_{2}, y\right)+\frac{1}{2 \pi i} \oint_{\mathcal{C}_{2}\left(u_{2}\right)} S^{\left(g_{1}+g_{2}\right)}\left(z_{2}, u_{2}\right) S^{\left(g_{1}\right)}\left(u_{2}, y\right)\right) \\
= & \xi \sum_{k \geq 1} h_{1}(k, x) \bar{h}_{2}(k, y) \\
& +\xi^{2} \sum_{k, l \geq 1} h_{1}(k, x) \bar{h}_{2}(l, y) \frac{\epsilon^{\frac{1}{2}(k+l-1)}}{(2 \pi i)^{2}} \oint_{\mathcal{C}_{2}\left(z_{2}\right)} \oint_{\mathcal{C}_{1}\left(u_{1}\right)} S^{\left(g_{1}+g_{2}\right)}\left(z_{2}, u_{1}\right) z_{2}^{-k} d z_{2}^{\frac{1}{2}} u_{1}^{-l} d u_{1}^{\frac{1}{2}} \\
= & h_{1}(x)\left(\xi I-X_{21}\right) \bar{h}_{2}^{T}(y) .
\end{aligned}
$$

A similar result holds for $x \in \widehat{\Sigma}^{\left(g_{2}\right)}, y \in \widehat{\Sigma}^{\left(g_{1}\right)}$.

We next compute the explicit form of the moment matrix $X_{a b}$ in terms of the moments $F_{a}$ of $S^{\left(g_{a}\right)}(x, y)$. It is useful to introduce infinite block matrices

$$
\begin{array}{ll}
X=\left(\begin{array}{cc}
X_{11} & X_{12} \\
X_{21} & X_{22}
\end{array}\right), & F=\left(\begin{array}{cc}
F_{1} & 0 \\
0 & F_{2}
\end{array}\right), \\
\Xi=\left(\begin{array}{cc}
0 & \xi I \\
-\xi I & 0
\end{array}\right), & Q=F \Xi=\left(\begin{array}{cc}
0 & \xi F_{1} \\
-\xi F_{2} & 0
\end{array}\right) .
\end{array}
$$

Then one finds:

Proposition $3.5 X$ is given by

$$
X=(I-Q)^{-1} F,
$$

where $(I-Q)^{-1}=\sum_{n \geq 0} Q^{n}$ is convergent for $|\epsilon|<\left|r_{1} r_{2}\right|$. 
Proof. Using (38) we find $X_{11}(k, l)-F_{1}(k, l)$ is given by

$$
\begin{aligned}
& -\frac{\epsilon^{\frac{1}{2}(k+l-1)}}{(2 \pi i)^{3}} \oint_{\mathcal{C}_{1}(x)} \oint_{\mathcal{C}_{1}\left(z_{1}\right)} S^{\left(g_{1}\right)}\left(x, z_{1}\right) x^{-k} d x^{\frac{1}{2}} \oint_{\mathcal{C}_{1}(y)} S^{\left(g_{1}+g_{2}\right)}\left(z_{1}, y\right) y^{-l} d y^{\frac{1}{2}} \\
& =-\frac{\epsilon^{\frac{1}{2}(k+l-1)}}{(2 \pi i)^{3}} \sum_{m \geq 0}\left(\epsilon^{-\frac{m}{2}+\frac{1}{4}} \oint_{\mathcal{C}_{1}(x)} h_{1}(m, x) x^{-k} d x^{\frac{1}{2}}\right. \\
& \left.\cdot \oint_{\mathcal{C}_{1}\left(z_{1}\right) \mathcal{C}_{1}(y)} S^{\left(g_{1}+g_{2}\right)}\left(z_{1}, y\right) z_{1}^{m-1} y^{-l} d y^{\frac{1}{2}}\right) \\
& =\xi \sum_{m \geq 0}\left(\frac{\epsilon^{\frac{k}{2}-\frac{1}{4}}}{2 \pi i} \oint_{\mathcal{C}_{1}(x)} h_{1}(m, x) x^{-k} d x^{\frac{1}{2}}\right. \\
& \left.\cdot \frac{\epsilon^{\frac{1}{2}(m+l-1)}}{(2 \pi i)^{2}} \oint_{\mathcal{C}_{2}\left(z_{2}\right)} \oint_{\mathcal{C}_{1}(y)} S^{\left(g_{1}+g_{2}\right)}\left(z_{2}, y\right) z_{2}^{-m} y^{-l} d y^{\frac{1}{2}}\right) \\
& =\xi \xi\left(F_{1} X_{21}\right)(k, l)
\end{aligned}
$$

using (47) and (49). Similarly we find $X_{22}=F_{2}-\xi F_{2} X_{12}$ so that $X_{a a}=$ $(F+Q X)_{a a}$ using (51). A similar calculation of $X_{12}$ and $X_{21}$ leads to $X_{a \bar{a}}=$ $(Q X)_{a \bar{a}}$. These combine to give $(I-Q) X=F$ which implies (52) provided $(I-Q)^{-1}=\sum_{n \geq 0} Q^{n}$ converges. But (52) can be rewritten

$$
X=\sum_{n \geq 1} Q^{n} \Xi
$$

By Theorem 3.2, $X_{a b}(k, l)$ has a convergent series expansion in $\epsilon^{\frac{1}{2}}$ for $|\epsilon|<$ $r_{1} r_{2}$. But $\sum_{n=1}^{N} Q^{n}=O\left(\epsilon^{\frac{1}{2} N}\right)$ so that (153) holds to all orders in $\epsilon^{\frac{1}{2}}$. Hence $(I-Q)^{-1}$ converges for $|\epsilon|<r_{1} r_{2}$ and the proposition holds.

Propositions 3.4 and 3.5 imply

Theorem $3.6 S^{\left(g_{1}+g_{2}\right)}(x, y)$ is given by

$$
S^{\left(g_{1}+g_{2}\right)}(x, y)=\delta_{a b} S^{\left(g_{a}\right)}(x, y)+h_{a}(x)\left(\Xi(I-Q)^{-1}\right)_{a b} \bar{h}_{b}^{T}(y),
$$


for $x \in \widehat{\Sigma}^{\left(g_{a}\right)}, y \in \widehat{\Sigma}^{\left(g_{b}\right)}$. Equivalently, $S^{\left(g_{1}+g_{2}\right)}(x, y)=\left\{\begin{array}{l}S^{\left(g_{a}\right)}(x, y)+h_{a}(x)\left(I-F_{\bar{a}} F_{a}\right)^{-1} F_{\bar{a}} \bar{h}_{a}^{T}(y), \quad \text { for } x, y \in \widehat{\Sigma}^{\left(g_{a}\right)}, \\ \xi(-1)^{\bar{a}} h_{a}(x)\left(I-F_{\bar{a}} F_{a}\right)^{-1} \bar{h}_{\bar{a}}^{T}(y), \quad \text { for } x \in \widehat{\Sigma}^{\left(g_{a}\right)}, y \in \widehat{\Sigma}^{\left(g_{\bar{a}}\right)}\end{array}\right.$

Remark 3.7 Note that $S^{\left(g_{1}+g_{2}\right)}(x, y)$ is even (odd) in $\epsilon^{\frac{1}{2}}$ for $x, y \in \widehat{\Sigma}^{\left(g_{a}\right)}$ (respectively, for $\left.x \in \widehat{\Sigma}^{\left(g_{a}\right)}, y \in \widehat{\Sigma}^{\left(g_{\bar{a}}\right)}\right)$. Thus $S^{\left(g_{1}+g_{2}\right)}(x, y)$ is invariant under a Dehn twist $\epsilon \rightarrow e^{2 \pi i} \epsilon$ with $\xi \rightarrow-\xi$ from (49).

Similarly to ref. MT1 we define the determinant of $I-Q$ as a formal power series in $\epsilon^{\frac{1}{2}}$ by

$$
\log \operatorname{det}(I-Q)=\operatorname{Tr} \log (I-Q)=-\sum_{n \geq 1} \frac{1}{n} \operatorname{Tr}\left(Q^{n}\right) .
$$

Clearly $\operatorname{Tr}\left(Q^{2 k}\right)=2 \operatorname{Tr}\left(\left(F_{1} F_{2}\right)^{k}\right)$ for $k \geq 0$ whereas $\operatorname{Tr}\left(Q^{n}\right)=0$ for $n$ odd. Furthermore, from (45) the diagonal terms $\left(F_{1} F_{2}\right)^{k}$ have integral power series in $\epsilon$. Thus it follows that

Lemma $3.8 \operatorname{det}(I-Q)=\operatorname{det}\left(I-F_{1} F_{2}\right)$ and is a formal power series in $\epsilon$.

The determinant has the following holomorphic properties:

Theorem $3.9 \operatorname{det}(I-Q)$ is non-vanishing and holomorphic in $\epsilon$ for $|\epsilon|<$ $r_{1} r_{2}$.

Proof. The proof follows a similar argument to Theorem 2 of ref. [MT1]. Let $S^{\left(g_{1}+g_{2}\right)}\left(z_{1}, z_{2}\right)=f\left(z_{1}, z_{2}, \epsilon\right) d z_{1}^{\frac{1}{2}} d z_{2}^{\frac{1}{2}}$ for $\left|z_{a}\right| \leq r_{a}$ where $f\left(z_{1}, z_{2}, \epsilon\right)$ is holomorphic in $\epsilon^{\frac{1}{2}}$ for $|\epsilon| \leq r$ for $r<r_{1} r_{2}$ from Theorem 3.2. Apply Cauchy's inequality to the coefficients of $f\left(z_{1}, z_{2}, \epsilon\right)=\sum_{n \geq 0} f_{n}\left(z_{1}, z_{2}\right) \epsilon^{\frac{n}{2}}$ to find

$$
\left|f_{n}\left(z_{1}, z_{2}\right)\right| \leq M r^{-\frac{n}{2}}
$$

for $M=\sup _{\left|z_{a}\right| \leq r_{a},|\epsilon| \leq r}\left|f\left(z_{1}, z_{2}, \epsilon\right)\right|$. Consider

$$
\mathcal{I}=\frac{1}{(2 \pi i)^{2}} \oint_{\mathcal{C}_{r_{1}}\left(z_{1}\right)} \oint_{\mathcal{C}_{r_{2}}\left(z_{2}\right)} S^{\left(g_{1}+g_{2}\right)}\left(z_{1}, z_{2}\right)\left(1-\frac{\epsilon}{z_{1} z_{2}}\right)^{-1} d z_{1}^{\frac{1}{2}} d z_{2}^{\frac{1}{2}}
$$


for $\mathcal{C}_{r_{a}}\left(z_{a}\right)$ the contour with $\left|z_{a}\right|=r_{a}$. Then using (54) we find

$$
|\mathcal{I}| \leq M \cdot \sum_{n \geq 0}\left(\frac{|\epsilon|}{r}\right)^{\frac{n}{2}} \cdot\left|1-\frac{|\epsilon|}{r_{1} r_{2}}\right|^{-1} \cdot r_{1} r_{2}
$$

i.e. $\mathcal{I}$ is absolutely convergent and thus holomorphic in $\epsilon^{\frac{1}{2}}$ for $|\epsilon|<r<r_{1} r_{2}$. Since $\left|z_{1} z_{2}\right|=r_{1} r_{2}$ we may alternatively expand in $\epsilon / z_{1} z_{2}$ to obtain

$$
\begin{aligned}
\mathcal{I} & =\sum_{k \geq 1} \epsilon^{k} \frac{1}{(2 \pi i)^{2}} \oint_{\mathcal{C}_{r_{1}}\left(z_{1}\right)} \oint_{\mathcal{C}_{r_{2}}\left(z_{2}\right)} S^{\left(g_{1}+g_{2}\right)}\left(z_{1}, z_{2}\right) z_{1}^{-k} z_{2}^{-k} d z_{1}^{\frac{1}{2}} d z_{2}^{\frac{1}{2}} \\
& =\epsilon^{\frac{1}{2}} \operatorname{Tr} X_{12}
\end{aligned}
$$

where $\operatorname{Tr} X_{12}=\sum_{k \geq 1} X_{12}(k, k)$. But (52) implies

$$
\operatorname{Tr} X_{12}=\xi \sum_{n \geq 1} \operatorname{Tr}\left(\left(F_{1} F_{2}\right)^{n}\right)
$$

which is absolutely convergent for $|\epsilon|<r_{1} r_{2}$. Hence we find

$$
\operatorname{Tr} \log \left(I-F_{1} F_{2}\right)=-\sum_{n \geq 1} \frac{1}{n} \operatorname{Tr}\left(\left(F_{1} F_{2}\right)^{n}\right),
$$

is also absolutely convergent for $|\epsilon|<r_{1} r_{2}$. Thus $\operatorname{det}(I-Q)=\operatorname{det}\left(I-F_{1} F_{2}\right)$ is non-vanishing and holomorphic for $|\epsilon|<r_{1} r_{2}$.

\subsection{Sewing Two Tori}

Consider the genus two surface formed by sewing two oriented tori $\Sigma_{a}^{(1)}=$ $\mathbb{C} / \Lambda_{a}$ for $a=1,2$, and lattice $\Lambda_{a}=2 \pi i\left(\mathbb{Z} \tau_{a} \oplus \mathbb{Z}\right)$ for $\tau_{a} \in \mathbb{H}_{1}$. This is discussed at length in [MT1]. For local coordinate $z_{a} \in \mathbb{C} / \Lambda_{a}$ consider the closed disk $\left|z_{a}\right| \leq r_{a}$ which is contained in $\Sigma_{a}^{(1)}$ provided $r_{a}<\frac{1}{2} D\left(q_{a}\right)$ where

$$
D\left(q_{a}\right)=\min _{\lambda \in \Lambda_{a}, \lambda \neq 0}|\lambda|
$$

is the minimal lattice distance. From Subsection 3.1 we obtain a genus two Riemann surface $\Sigma^{(2)}$ parameterized by the domain

$$
\mathcal{D}^{\epsilon}=\left\{\left(\tau_{1}, \tau_{2}, \epsilon\right) \in \mathbb{H}_{1} \times \mathbb{H}_{1} \times \mathbb{C}:|\epsilon|<\frac{1}{4} D\left(q_{1}\right) D\left(q_{2}\right)\right\} .
$$


$\mathcal{D}^{\epsilon}$ is preserved under the action of $G \simeq(S L(2, \mathbb{Z}) \times S L(2, \mathbb{Z})) \rtimes \mathbb{Z}_{2}$, the direct product of the left and right torus modular groups, which are interchanged upon conjugation by an involution $\beta$ as follows

$$
\begin{aligned}
\gamma_{1}\left(\tau_{1}, \tau_{2}, \epsilon\right) & =\left(\frac{a_{1} \tau_{1}+b_{1}}{c_{1} \tau_{1}+d_{1}}, \tau_{2}, \frac{\epsilon}{c_{1} \tau_{1}+d_{1}}\right) \\
\gamma_{2}\left(\tau_{1}, \tau_{2}, \epsilon\right) & =\left(\tau_{1}, \frac{a_{2} \tau_{2}+b_{2}}{c_{2} \tau_{2}+d_{2}}, \frac{\epsilon}{c_{2} \tau_{2}+d_{2}}\right) \\
\beta\left(\tau_{1}, \tau_{2}, \epsilon\right) & =\left(\tau_{2}, \tau_{1}, \epsilon\right),
\end{aligned}
$$

for $\left(\gamma_{1}, \gamma_{2}\right) \in S L(2, \mathbb{Z}) \times S L(2, \mathbb{Z})$ with $\gamma_{i}=\left(\begin{array}{ll}a_{i} & b_{i} \\ c_{i} & d_{i}\end{array}\right)$.

There is a natural injection $G \rightarrow S p(4, \mathbb{Z})$ in which the two $S L(2, \mathbb{Z})$ subgroups are mapped to

$$
\Gamma_{1}=\left\{\left[\begin{array}{cccc}
a_{1} & 0 & b_{1} & 0 \\
0 & 1 & 0 & 0 \\
c_{1} & 0 & d_{1} & 0 \\
0 & 0 & 0 & 1
\end{array}\right]\right\}, \Gamma_{2}=\left\{\left[\begin{array}{cccc}
1 & 0 & 0 & 0 \\
0 & a_{2} & 0 & b_{2} \\
0 & 0 & 1 & 0 \\
0 & c_{2} & 0 & d_{2}
\end{array}\right]\right\},
$$

and the involution is mapped to

$$
\beta=\left[\begin{array}{llll}
0 & 1 & 0 & 0 \\
1 & 0 & 0 & 0 \\
0 & 0 & 0 & 1 \\
0 & 0 & 1 & 0
\end{array}\right]
$$

$G$ also has a natural action on $\mathbb{H}_{2}$ as given in (5) which is compatible with respect to $\Omega^{(2)}$ as a function of $\left(\tau_{1}, \tau_{2}, \epsilon\right)$ [MT1.

The Szegö kernel on the torus $\Sigma_{a}^{(1)}$ is given by

$$
S^{(1)}\left[\begin{array}{l}
\theta_{a} \\
\phi_{a}
\end{array}\right]\left(x, y \mid \tau_{a}\right)=P_{1}\left[\begin{array}{l}
\theta_{a} \\
\phi_{a}
\end{array}\right]\left(x-y, \tau_{a}\right) d x^{\frac{1}{2}} d y^{\frac{1}{2}},
$$

from (23). It is straightforward to compute the moment matrix $F_{a}$ of (45). Using the Laurant expansion (28) we find

$$
P_{1}\left[\begin{array}{l}
\theta \\
\phi
\end{array}\right](x-y, \tau)=\frac{1}{x-y}+\sum_{k, l \geq 1} C\left[\begin{array}{l}
\theta \\
\phi
\end{array}\right](k, l) x^{k-1} y^{l-1},
$$


where for $k, l \geq 1$ we define

$$
C\left[\begin{array}{l}
\theta \\
\phi
\end{array}\right](k, l, \tau)=(-1)^{l}\left(\begin{array}{c}
k+l-2 \\
k-1
\end{array}\right) E_{k+l-1}\left[\begin{array}{l}
\theta \\
\phi
\end{array}\right](\tau),
$$

for twisted Eisenstein series (29). Then it follows that

$$
F_{a}\left[\begin{array}{l}
\theta_{a} \\
\phi_{a}
\end{array}\right]\left(k, l, \tau_{a}, \epsilon\right)=\epsilon^{\frac{1}{2}(k+l-1)} C\left[\begin{array}{l}
\theta_{a} \\
\phi_{a}
\end{array}\right]\left(k, l, \tau_{a}\right) .
$$

We also have the analytic expansion

$$
P_{1}\left[\begin{array}{l}
\theta \\
\phi
\end{array}\right](x-y, \tau)=\sum_{k \geq 0} P_{k}\left[\begin{array}{l}
\theta \\
\phi
\end{array}\right](x, \tau) y^{k-1},
$$

for $P_{k}\left[\begin{array}{l}\theta \\ \phi\end{array}\right](z, \tau)=\frac{(-1)^{k-1}}{(k-1) !} \partial_{z}^{k-1} P_{1}\left[\begin{array}{l}\theta \\ \phi\end{array}\right](z, \tau)$. Then we find

$$
h_{a}\left[\begin{array}{l}
\theta_{a} \\
\phi_{a}
\end{array}\right]\left(k, x, \tau_{a}, \epsilon\right)=\epsilon^{\frac{k}{2}-\frac{1}{4}} P_{k}\left[\begin{array}{l}
\theta_{a} \\
\phi_{a}
\end{array}\right]\left(x, \tau_{a}\right) d x^{\frac{1}{2}} .
$$

Using these results we may therefore determine the explicit form for $S^{(2)}\left[\begin{array}{l}\theta^{(2)} \\ \phi^{(2)}\end{array}\right]$ on $\mathcal{D}^{\epsilon}$ via Theorem 3.6.

One may also confirm that $S^{(2)}$ satisfies the modular invariance property of (201) under the group $G$ generated by $\gamma_{i}, \beta$ of (58) and (59) with

$$
S^{(2)}\left(\gamma\left[\begin{array}{l}
\theta^{(2)} \\
\phi^{(2)}
\end{array}\right]\right)\left(\gamma x, \gamma y \mid \gamma\left(\tau_{1}, \tau_{2}, \epsilon\right)\right)=S^{(2)}\left[\begin{array}{l}
\theta^{(2)} \\
\phi^{(2)}
\end{array}\right]\left(x, y \mid \tau_{1}, \tau_{2}, \epsilon\right),
$$

where

$\gamma_{1}\left[\begin{array}{c}\theta_{1} \\ \theta_{2} \\ \phi_{1} \\ \phi_{2}\end{array}\right]=\left[\begin{array}{c}\theta_{1}^{a_{1}} \phi_{1}^{b_{1}} \\ \theta_{2} \\ \theta_{1}^{c_{1}} \phi_{1}^{d_{1}} \\ \phi_{2}\end{array}\right], \quad \gamma_{2}\left[\begin{array}{c}\theta_{1} \\ \theta_{2} \\ \phi_{1} \\ \phi_{2}\end{array}\right]=\left[\begin{array}{c}\theta_{1} \\ \theta_{2}^{a_{2}} \phi_{2}^{b_{2}} \\ \phi_{1} \\ \theta_{2}^{c_{2}} \phi_{2}^{d_{2}}\end{array}\right], \quad \beta\left[\begin{array}{c}\theta_{1} \\ \theta_{2} \\ \phi_{1} \\ \phi_{2}\end{array}\right]=\left[\begin{array}{c}\theta_{2} \\ \theta_{1} \\ \phi_{2} \\ \phi_{1}\end{array}\right]$,

and

$$
\gamma_{a} x=\left\{\begin{array}{cl}
\frac{x}{c_{a} \tau_{a}+d_{a}}, & \text { for } x \in \widehat{\Sigma}_{a}^{(1)} \\
x, & \text { for } x \in \widehat{\Sigma}_{\bar{a}}^{(1)}
\end{array}\right.
$$

and where for $x=2 \pi i\left(u+v \tau_{a}\right) \in \widehat{\Sigma}_{a}^{(1)}$ with $0 \leq u, v<1$ we define $\beta x=$ $2 \pi i\left(u+v \tau_{\bar{a}}\right)$. Finally, we note that $\operatorname{det}(I-Q)=\operatorname{det}\left(I-F_{1}\left[\begin{array}{l}\theta_{1} \\ \phi_{1}\end{array}\right] F_{2}\left[\begin{array}{l}\theta_{2} \\ \phi_{2}\end{array}\right]\right)$ is also $G$ invariant. 


\section{The Szegö kernel on a Self-Sewn Riemann Surface}

\subsection{The $\rho$-Formalism Sewing Scheme}

We now consider the construction of the Szegö kernel on a Riemann surface $\Sigma^{(g+1)}$ formed by self-sewing a handle to a Riemann surface $\Sigma^{(g)}$ of genus $g$. We begin by reviewing the Yamada formalism [Y] in this scheme which, following [MT1], we refer to as the $\rho$-formalism. Consider a Riemann surface $\Sigma^{(g)}$ of genus $g$ and let $z_{1}, z_{2}$ be local coordinates in the neighborhood of two separated points $p_{1}$ and $p_{2}$. Consider two disks $\left|z_{a}\right| \leq r_{a}$, for $r_{a}>0$ and $a=1,2$. Note that $r_{1}, r_{2}$ must be sufficiently small to ensure that the disks do not intersect. Introduce a complex parameter $\rho$ where $|\rho| \leq r_{1} r_{2}$ and excise the disks

$$
\left\{z_{a}:\left|z_{a}\right|<|\rho| r_{\bar{a}}^{-1}\right\} \subset \Sigma^{(g)},
$$

to form a twice-punctured surface

$$
\widehat{\Sigma}^{(g)}=\Sigma^{(g)} \backslash \bigcup_{a=1,2}\left\{z_{a}:\left|z_{a}\right|<|\rho| r_{\bar{a}}^{-1}\right\} .
$$

As before, we use the convention $\overline{1}=2, \overline{2}=1$. We define annular regions $\mathcal{A}_{a} \subset \widehat{\Sigma}^{(g)}$ with $\mathcal{A}_{a}=\left\{z_{a}:|\rho| r_{\bar{a}}^{-1} \leq\left|z_{a}\right| \leq r_{a}\right\}$ and identify them as a single region $\mathcal{A}=\mathcal{A}_{1} \simeq \mathcal{A}_{2}$ via the sewing relation

$$
z_{1} z_{2}=\rho
$$

to form a compact Riemann surface $\Sigma^{(g+1)}=\widehat{\Sigma}^{(g)} \backslash\left\{\mathcal{A}_{1} \cup \mathcal{A}_{2}\right\} \cup \mathcal{A}$ of genus $g+1$. The sewing relation (66) can be considered to be a parameterization of a cylinder connecting the punctured Riemann surface to itself.

In the $\rho$-formalism we define a standard basis of cycles $\left\{a_{1}, b_{1}, \ldots, a_{g+1}, b_{g+1}\right\}$ on $\Sigma^{(g+1)}$ where the set $\left\{a_{1}, b_{1}, \ldots, a_{g}, b_{g}\right\}$ is the original basis on $\Sigma^{(g)}$. Let $\mathcal{C}_{a}\left(z_{a}\right) \subset \mathcal{A}_{a}$ denote a closed anti-clockwise contour parameterized by $z_{a}$ surrounding the puncture at $z_{a}=0$. Clearly $\mathcal{C}_{2}\left(z_{2}\right) \sim-\mathcal{C}_{1}\left(z_{1}\right)$ on applying the sewing relation (66). We then define the cycle $a_{g+1}$ to be $\mathcal{C}_{2}\left(z_{2}\right)$ and define the cycle $b_{g+1}$ to be a path chosen in $\widehat{\Sigma}^{(g)}$ between identified points $z_{1}=z_{0}$ and $z_{2}=\rho / z_{0}$ on the sewn surface.

As in the $\epsilon$-formalism, the normalized differential of the second kind $\omega^{(g+1)}$, the holomorphic 1 -forms $\nu_{i}^{(g+1)}$ and the period matrix $\Omega^{(g+1)}$ can be computed in terms of data coming from $\Sigma^{(g)}[\mathrm{Y}]$, [MT1 to find 
Theorem $4.1 \omega^{(g+1)}, \nu_{i}^{(g+1)}$ and $\Omega_{i j}^{(g+1)}$ for $(i, j) \neq(g+1, g+1)$ are holomorphic in $\rho$ for $|\rho|<r_{1} r_{2}$ with

$$
\begin{aligned}
& \omega^{(g+1)}(x, y)=\omega^{(g)}(x, y)+O(\rho), \\
& \nu_{i}^{(g+1)}(x)=\nu_{i}^{(g)}(x)+O(\rho), \quad i=1, \ldots, g \\
& \nu_{g+1}^{(g+1)}(x)=\omega_{p_{2}-p_{1}}^{(g)}(x)+O(\rho), \\
& \Omega_{i j}^{(g+1)}=\Omega_{i j}^{(g)}+O(\rho), \quad i, j=1, \ldots, g \\
& \Omega_{i, g+1}^{(g+1)}=\frac{1}{2 \pi i} \int_{p_{1}}^{p_{2}} \nu_{i}^{(g)}+O(\rho), \quad i=1, \ldots, g,
\end{aligned}
$$

for $x, y \in \widehat{\Sigma}^{(g)} \cdot e^{2 \pi i \Omega_{g+1, g+1}^{(g+1)}}$ is holomorphic in $\rho$ for $|\rho|<r_{1} r_{2}$ with

$$
e^{2 \pi i \Omega_{g+1, g+1}^{(g+1)}}=-\frac{\rho}{K_{0}^{2}}(1+O(\rho))
$$

where $K_{0}=K^{(g)}\left(z_{1}=0, z_{2}=0\right)$ for $E^{(g)}\left(z_{1}, z_{2}\right)=K^{(g)}\left(z_{1}, z_{2}\right) d z_{1}^{-\frac{1}{2}} d z_{2}^{-\frac{1}{2}}$ expressed in terms of the local coordinates $z_{1}, z_{2}$.

\subsection{Szegö Kernel in the $\rho$-Formalism}

We now determine the Szegö kernel $S^{(g+1)}(x, y)=S^{(g+1)}\left[\begin{array}{l}\theta^{(g+1)} \\ \phi^{(g+1)}\end{array}\right](x, y)$ on the sewn Riemann surface $\Sigma^{(g+1)}$ in terms of genus $g$ data together with the multiplier parameters associated with the handle cycles. The $S^{(g+1)}$ multipliers (17) on the cycles $a_{i}, b_{i}$ for $i=1, \ldots, g$ are determined by the multipliers of $S^{(g)}$ with $\phi_{i}^{(g+1)}=\phi_{i}^{(g)}$ and $\theta_{i}^{(g+1)}=\theta_{i}^{(g)}$ i.e. $\alpha_{i}^{(g+1)}=\alpha_{i}^{(g)}$ and $\beta_{i}^{(g+1)}=\beta_{i}^{(g)}$. The remaining two multipliers associated with the cycles $a_{g+1}$ and $b_{g+1}$

$$
\begin{aligned}
& \phi_{g+1}=\phi_{g+1}^{(g+1)}=-e^{2 \pi i \alpha_{g+1}^{(g+1)}}, \\
& \theta_{g+1}=\theta_{g+1}^{(g+1)}=-e^{-2 \pi i \beta_{g+1}^{(g+1)}},
\end{aligned}
$$

must be additionally specified so that

$$
\begin{aligned}
S^{(g+1)}\left(e^{2 \pi i} x_{a}, y\right) & =-\phi_{g+1}^{a-\bar{a}} S^{(g+1)}\left(x_{a}, y\right), \\
S^{(g+1)}\left(x_{a}, y\right) & =-\theta_{g+1}^{a-\bar{a}} S^{(g+1)}\left(x_{\bar{a}}, y\right),
\end{aligned}
$$

for $x_{a} \in \mathcal{A}_{a}$ and $x_{\bar{a}} \in \mathcal{A}_{\bar{a}}$. 
We next consider the analogue of Theorem 3.2 concerning the holomorphicity of $S^{(g+1)}$ as a function of $\rho$. It is convenient to define $\kappa \in\left[-\frac{1}{2}, \frac{1}{2}\right)$ by $\phi_{g+1}=-e^{2 \pi i \kappa}$ i.e. $\kappa=\alpha_{g+1}^{(g+1)} \bmod 1$. We then find

Theorem 4.2 $S^{(g+1)}$ is holomorphic in $\rho$ for $|\rho|<r_{1} r_{2}$ with

$$
S^{(g+1)}(x, y)=S_{\kappa}^{(g)}(x, y)+O(\rho)
$$

for $x, y \in \widehat{\Sigma}^{(g)}$ where $S_{\kappa}^{(g)}(x, y)$ is defined as follows: For $\kappa \neq-\frac{1}{2}$

$$
S_{\kappa}^{(g)}(x, y)=\frac{U(x, y)^{\kappa} \vartheta\left[\begin{array}{l}
\alpha^{(g)} \\
\beta^{(g)}
\end{array}\right]\left(\int_{y}^{x} \nu^{(g)}+\kappa z_{p_{1}, p_{2}} \mid \Omega^{(g)}\right)}{E^{(g)}(x, y) \vartheta\left[\begin{array}{l}
\alpha^{(g)} \\
\beta^{(g)}
\end{array}\right]\left(\kappa z_{p_{1}, p_{2}} \mid \Omega^{(g)}\right)}
$$

where

$$
U(x, y)=\frac{E^{(g)}\left(x, p_{2}\right) E^{(g)}\left(y, p_{1}\right)}{E^{(g)}\left(x, p_{1}\right) E^{(g)}\left(y, p_{2}\right)},
$$

for prime form $E^{(g)}$ and where

$$
z_{p_{1}, p_{2}}=\int_{p_{1}}^{p_{2}} \nu^{(g)}
$$

for holomorphic 1-forms $\nu^{(g)}$. For $\kappa=-\frac{1}{2}$ then $S_{-\frac{1}{2}}^{(g)}(x, y)$ is given by

$$
\begin{gathered}
\left(\frac{U(x, y)^{\frac{1}{2}}}{E^{(g)}(x, y)} \vartheta\left[\begin{array}{l}
\alpha^{(g)} \\
\beta^{(g)}
\end{array}\right]\left(\int_{y}^{x} \nu^{(g)}+\frac{1}{2} z_{p_{1}, p_{2}} \mid \Omega^{(g)}\right)\right. \\
\left.-\theta_{g+1} \frac{U(x, y)^{-\frac{1}{2}}}{E^{(g)}(x, y)} \vartheta\left[\begin{array}{l}
\alpha^{(g)} \\
\beta^{(g)}
\end{array}\right]\left(\int_{y}^{x} \nu^{(g)}-\frac{1}{2} z_{p_{1}, p_{2}} \mid \Omega^{(g)}\right)\right) . \\
\left(\vartheta\left[\begin{array}{c}
\alpha^{(g)} \\
\beta^{(g)}
\end{array}\right]\left(\frac{1}{2} z_{p_{1}, p_{2}} \mid \Omega^{(g)}\right)-\theta_{g+1} \vartheta\left[\begin{array}{l}
\alpha^{(g)} \\
\beta^{(g)}
\end{array}\right]\left(-\frac{1}{2} z_{p_{1}, p_{2}} \mid \Omega^{(g)}\right)\right)^{-1} .
\end{gathered}
$$

Proof. We firstly note that from (15) it follows that

$$
E^{(g+1)}(x, y)=E^{(g)}(x, y)+O(\rho) .
$$

From Theorem 4.1 we may expand the genus $g+1$ theta series to leading 
order in $\rho$ for $|\rho|<r_{1} r_{2}$ as follows

$$
\begin{aligned}
& \vartheta\left[\begin{array}{l}
\alpha^{(g+1)} \\
\beta^{(g+1)}
\end{array}\right]\left(\int_{y}^{x} \nu^{(g+1)} \mid \Omega^{(g+1)}\right)=\sum_{m \in \mathbb{Z}^{g}} \sum_{n \in \mathbb{Z}}\left(-\frac{\rho}{K_{0}^{2}}\right)^{\frac{1}{2}\left(n+\alpha_{g+1}^{(g+1)}\right)^{2}} \cdot \\
& \exp \left(i \pi\left(m+\alpha^{(g)}\right) \cdot \Omega^{(g)} \cdot\left(m+\alpha^{(g)}\right)+\left(m+\alpha^{(g)}\right) \cdot\left(\int_{y}^{x} \nu^{(g)}+2 \pi i \beta^{(g)}\right)+\right. \\
& \left.\left(n+\alpha_{g+1}^{(g+1)}\right)\left[\left(m+\alpha^{(g)}\right) \cdot \int_{p_{1}}^{p_{2}} \nu^{(g)}+\int_{y}^{x} \omega_{p_{2}-p_{1}}^{(g)}+2 \pi i \beta_{g+1}^{(g+1)}\right]\right) . \\
& (1+O(\rho))
\end{aligned}
$$

Clearly $\left|n+\alpha_{g+1}^{(g+1)}\right| \geq|\kappa|$. For $\kappa \neq-\frac{1}{2}$ it follows that this lower bound is satisfied for one value of $n$ so that

$$
\begin{aligned}
& \vartheta\left[\begin{array}{l}
\alpha^{(g+1)} \\
\beta^{(g+1)}
\end{array}\right]\left(\int_{y}^{x} \nu^{(g+1)} \mid \Omega^{(g+1)}\right)=\left(-\frac{\rho}{K_{0}^{2}}\right)^{\frac{1}{2} \kappa^{2}}\left(-\theta_{g+1}\right)^{-\kappa} U(x, y)^{\kappa} \\
& \sum_{m \in \mathbb{Z}^{g}} \exp \left(i \pi\left(m+\alpha^{(g)}\right) \cdot \Omega^{(g)} \cdot\left(m+\alpha^{(g)}\right)\right. \\
& \left.+\left(m+\alpha^{(g)}\right) \cdot\left(\int_{y}^{x} \nu^{(g)}+\kappa z_{p_{1}, p_{2}}+2 \pi i \beta^{(g)}\right)\right) \cdot(1+O(\rho)),
\end{aligned}
$$

for $z_{p_{1}, p_{2}}$ of (80) and where from (13)

$$
\int_{y}^{x} \omega_{p_{2}-p_{1}}^{(g)}=\int_{y}^{x} \int_{p_{1}}^{p_{2}} \omega^{(g)}(\cdot, \cdot)=\log U(x, y),
$$

for $U(x, y)$ of (179). Therefore

$$
\begin{aligned}
& \vartheta\left[\begin{array}{l}
\alpha^{(g+1)} \\
\beta^{(g+1)}
\end{array}\right]\left(\int_{y}^{x} \nu^{(g+1)} \mid \Omega^{(g+1)}\right)= \\
& \left(-\frac{\rho}{K_{0}^{2}}\right)^{\frac{1}{2} \kappa^{2}}\left(-\theta_{g+1}\right)^{-\kappa} U(x, y)^{\kappa} \vartheta\left[\begin{array}{l}
\alpha^{(g)} \\
\beta^{(g)}
\end{array}\right]\left(\int_{y}^{x} \nu^{(g)}+\kappa z_{p_{1}, p_{2}} \mid \Omega^{(g)}\right)(1+O(\rho)) .
\end{aligned}
$$

Since $U(x, x)=1$ we find that for $\kappa \neq-\frac{1}{2}$

$$
\frac{\vartheta\left[\begin{array}{l}
\alpha^{(g+1)} \\
\beta^{(g+1)}
\end{array}\right]\left(\int_{y}^{x} \nu^{(g+1)} \mid \Omega^{(g+1)}\right)}{\vartheta\left[\begin{array}{l}
\alpha^{(g+1)} \\
\beta^{(g+1)}
\end{array}\right]\left(0 \mid \Omega^{(g+1)}\right)}=U(x, y)^{\kappa} \frac{\vartheta\left[\begin{array}{l}
\alpha^{(g)} \\
\beta^{(g)}
\end{array}\right]\left(\int_{y}^{x} \nu^{(g)}+\kappa z_{p_{1}, p_{2}} \mid \Omega^{(g)}\right)}{\vartheta\left[\begin{array}{l}
\alpha^{(g)} \\
\beta^{(g)}
\end{array}\right]\left(\kappa z_{p_{1}, p_{2}} \mid \Omega^{(g)}\right)}(1+O(\rho)),
$$


is holomorphic in $\rho$ for $|\rho|<r_{1} r_{2}$. Combining this result with (82) we immediately find (178) using the definition of the Szegö kernel (16).

For $\kappa=-\frac{1}{2}$ the lower bound on $\left|n+\alpha_{g+1}^{(g+1)}\right|=|\kappa|$ is satisfied for two values of $n$ so that

$$
\begin{aligned}
& \vartheta\left[\begin{array}{l}
\alpha^{(g+1)} \\
\beta^{(g+1)}
\end{array}\right]\left(\int_{y}^{x} \nu^{(g+1)} \mid \Omega^{(g+1)}\right)= \\
& \left(-\frac{\rho}{K_{0}^{2}}\right)^{\frac{1}{8}}\left[\left(-\theta_{g+1}\right)^{-\frac{1}{2}} U(x, y)^{\frac{1}{2}} \vartheta\left[\begin{array}{c}
\alpha^{(g)} \\
\beta^{(g)}
\end{array}\right]\left(\int_{y}^{x} \nu^{(g)}+\frac{1}{2} z_{p_{1}, p_{2}} \mid \Omega^{(g)}\right)\right. \\
& \left.+\left(-\theta_{g+1}\right)^{\frac{1}{2}} U(x, y)^{-\frac{1}{2}} \vartheta\left[\begin{array}{c}
\alpha^{(g)} \\
\beta^{(g)}
\end{array}\right]\left(\int_{y}^{x} \nu^{(g)}-\frac{1}{2} z_{p_{1}, p_{2}} \mid \Omega^{(g)}\right)\right](1+O(\rho)) .
\end{aligned}
$$

which eventually leads to (81).

We next note that, similarly to (37), $S_{\kappa}^{(g)}\left(x, z_{a}\right) S^{(g+1)}\left(z_{a}, y\right)$ is a meromorphic 1 -form in $z_{a}$ periodic on the $\Sigma^{(g)}$ cycles $a_{i}, b_{i}$ for $i=1, \ldots, g$ with simple poles

$$
\begin{aligned}
S_{\kappa}^{(g)}\left(x, z_{a}\right) S^{(g+1)}\left(z_{a}, y\right) & \sim \frac{d z_{a}}{x-z_{a}} S^{(g+1)}(x, y) \text { for } z_{a} \sim x, \\
S_{\kappa}^{(g)}\left(x, z_{a}\right) S^{(g+1)}\left(z_{a}, y\right) & \sim \frac{d z_{a}}{z_{a}-y} S_{\kappa}^{(g)}(x, y) \text { for } z_{a} \sim y .
\end{aligned}
$$

Furthermore, $S_{\kappa}^{(g)}\left(x, z_{a}\right) S^{(g+1)}\left(z_{a}, y\right)$ is also periodic on the $a_{g+1}$ cycle defined by $\mathcal{C}_{2}\left(z_{2}\right) \sim-\mathcal{C}_{1}\left(z_{1}\right)$. This follows from applying (75) to (77) so that

$$
S_{\kappa}^{(g)}\left(x, e^{2 \pi i} z_{a}\right)=e^{2 \pi i \kappa(\bar{a}-a)} S_{\kappa}^{(g)}\left(x, z_{a}\right),
$$

(or alternatively we may apply $\left.U\left(x, e^{2 \pi i} z_{a}\right)^{\kappa}=e^{2 \pi i \kappa(\bar{a}-a)} U\left(x, z_{a}\right)^{\kappa}\right)$. Similar properties hold for $S_{\kappa}^{(g)}\left(x, z_{a}\right) S^{(g+1)}\left(z_{a}, y\right)$. This leads to the following analogue of Proposition 3.3

Proposition 4.3 The Szegö kernel on a genus $g+1$ Riemann surface in the $\rho$-formalism for $x, y \in \widehat{\Sigma}^{(g)}$ is given by

$$
\begin{aligned}
S^{(g+1)}(x, y) & =S_{\kappa}^{(g)}(x, y)+\sum_{a=1,2} \frac{1}{2 \pi i} \oint_{\mathcal{C}_{a}\left(z_{a}\right)} S_{\kappa}^{(g)}\left(x, z_{a}\right) S^{(g+1)}\left(z_{a}, y\right) \\
& =S_{\kappa}^{(g)}(x, y)-\sum_{a=1,2} \frac{1}{2 \pi i} \oint_{\mathcal{C}_{a}\left(z_{a}\right)} S^{(g+1)}\left(x, z_{a}\right) S_{\kappa}^{(g)}\left(z_{a}, y\right) .
\end{aligned}
$$


Proof. The proof follows along the same lines as Proposition 3.3. Let $\sigma$ be a contour on $\widehat{\Sigma}^{(g)}$ surrounding $\mathcal{A}_{a}$ and the given points $x, y \in \widehat{\Sigma}^{(g)}$ as shown in Fig. 3.

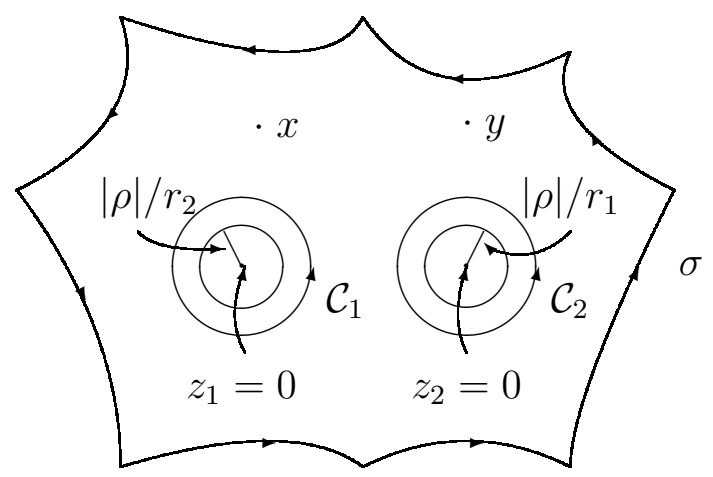

Fig. 3: Contour $\sigma$

Cauchy's Theorem and (83) imply

$$
\begin{aligned}
0 & =\frac{1}{2 \pi i} \oint_{\sigma} S_{\kappa}^{(g)}(x, z) S^{(g+1)}(z, y) \\
& =-S^{(g+1)}(x, y)+S_{\kappa}^{(g)}(x, y)+\sum_{a=1,2} \frac{1}{2 \pi i} \oint_{\mathcal{C}_{a}\left(z_{a}\right)} S_{\kappa}^{(g)}\left(x, z_{a}\right) S^{(g+1)}\left(z_{a}, y\right),
\end{aligned}
$$

recalling that $S_{\kappa}^{(g)}\left(x, z_{a}\right) S^{(g+1)}\left(z_{a}, y\right)$ is periodic on $\mathcal{C}_{a}$. Thus (85) follows. A similar argument holds for (86).

We next define weighted moments of $S^{(g+1)}(x, y)$. Let

$$
k_{a}=k+(-1)^{\bar{a}} \kappa,
$$

for $a=1,2$ and integer $k \geq 1$ and define

$$
\begin{aligned}
& Y_{a b}(k, l)=Y_{a b}\left[\begin{array}{c}
\theta^{(g+1)} \\
\phi^{(g+1)}
\end{array}\right](k, l) \\
& =\frac{\rho^{\frac{1}{2}\left(k_{a}+l_{b}-1\right)}}{(2 \pi i)^{2}} \oint_{\mathcal{C}_{\bar{a}}\left(x_{\bar{a}}\right)} \oint_{\mathcal{C}_{b}\left(y_{b}\right)}\left(x_{\bar{a}}\right)^{-k_{a}}\left(y_{b}\right)^{-l_{b}} S^{(g+1)}\left(x_{\bar{a}}, y_{b}\right) d x_{\bar{a}}^{\frac{1}{2}} d y_{b}^{\frac{1}{2}} .
\end{aligned}
$$

We define $Y=\left(Y_{a b}(k, l)\right)$ to be the infinite matrix indexed by $a, k$ and $b, l$. From (19) we note the skew-symmetry property

$$
Y_{a b}\left[\begin{array}{l}
\theta^{(g+1)} \\
\phi^{(g+1)}
\end{array}\right](k, l)=-Y_{\bar{b} \bar{a}}\left[\begin{array}{c}
\left(\theta^{(g+1)}\right)^{-1} \\
\left(\phi^{(g+1)}\right)^{-1}
\end{array}\right](k, l) .
$$


We also introduce moments for $S_{\kappa}^{(g)}(x, y)$

$$
\begin{aligned}
& G_{a b}(k, l)=G_{a b}\left[\begin{array}{l}
\theta^{(g)} \\
\phi^{(g)}
\end{array}\right](\kappa ; k, l) \\
& =\frac{\rho^{\frac{1}{2}\left(k_{a}+l_{b}-1\right)}}{(2 \pi i)^{2}} \oint_{\mathcal{C}_{\bar{a}\left(x_{\bar{a}}\right)}} \oint_{\mathcal{C}_{b}\left(y_{b}\right)}\left(x_{\bar{a}}\right)^{-k_{a}}\left(y_{b}\right)^{-l_{b}} S_{\kappa}^{(g)}\left(x_{\bar{a}}, y_{b}\right) d x_{\bar{a}}^{\frac{1}{2}} d y_{b}^{\frac{1}{2}},
\end{aligned}
$$

with associated infinite matrix $G=\left(G_{a b}(k, l)\right)$. This also satisfies a skewsymmetry property

$$
G_{a b}\left[\begin{array}{l}
\theta^{(g)} \\
\phi^{(g)}
\end{array}\right](\kappa ; k, l)=-G_{\bar{b} \bar{a}}\left[\begin{array}{c}
\left(\theta^{(g)}\right)^{-1} \\
\left(\phi^{(g)}\right)^{-1}
\end{array}\right](-\kappa ; k, l) .
$$

Finally we define half-order differentials

$$
\begin{aligned}
& h_{a}(k, x)=h_{a}\left[\begin{array}{l}
\theta^{(g)} \\
\phi^{(g)}
\end{array}\right](\kappa ; k, x)=\frac{\rho^{\frac{1}{2}\left(k_{a}-\frac{1}{2}\right)}}{2 \pi i} \oint_{\mathcal{C}_{a}\left(y_{a}\right)} y_{a}^{-k_{a}} S_{\kappa}^{(g)}\left(x, y_{a}\right) d y_{a}^{\frac{1}{2}}, \\
& \bar{h}_{a}(k, y)=\bar{h}_{a}\left[\begin{array}{l}
\theta^{(g)} \\
\phi^{(g)}
\end{array}\right](\kappa ; k, y)=\frac{\rho^{\frac{1}{2}\left(k_{a}-\frac{1}{2}\right)}}{2 \pi i} \oint_{\mathcal{C}_{\bar{a}\left(x_{\bar{a}}\right)}} x_{\bar{a}}^{-k_{a}} S_{\kappa}^{(g)}\left(x_{\bar{a}}, y\right) d x_{\bar{a}}^{\frac{1}{2}},
\end{aligned}
$$

and let $h(x)=\left(h_{a}(k, x)\right)$ and $\bar{h}(y)=\left(\bar{h}_{a}(k, y)\right)$ denote the infinite row vectors indexed by $a, k$. These are related by skew-symmetry with

$$
h_{a}\left[\begin{array}{l}
\theta^{(g)} \\
\phi^{(g)}
\end{array}\right](\kappa ; k, x)=-\bar{h}_{\bar{a}}\left[\begin{array}{c}
\left(\theta^{(g)}\right)^{-1} \\
\left(\phi^{(g)}\right)^{-1}
\end{array}\right](-\kappa ; k, x) .
$$

These moments can be inverted to obtain

$$
\begin{aligned}
& S_{\kappa}^{(g)}\left(x, y_{a}\right)=\sum_{k \geq 1} \rho^{-\frac{k_{a}}{2}+\frac{1}{4}} h_{a}(k, x) y_{a}^{k_{a}-1} d y_{a}^{\frac{1}{2}} \\
& S_{\kappa}^{(g)}\left(x_{\bar{a}}, y\right)=\sum_{k \geq 1} \rho^{-\frac{k_{a}}{2}+\frac{1}{4}} x_{\bar{a}}^{k_{a}-1} \bar{h}_{a}(k, y) d x_{\bar{a}}^{\frac{1}{2}} .
\end{aligned}
$$

From the sewing relation (66) we have

$$
d z_{a}^{\frac{1}{2}}=(-1)^{\bar{a}} \xi \rho^{\frac{1}{2}} \frac{d z_{\bar{a}}^{\frac{1}{2}}}{z_{\bar{a}}}
$$

for $\xi \in\{ \pm \sqrt{-1}\}$. We then find in a similar way to Proposition 3.4 that 
Proposition 4.4 For $x, y \in \widehat{\Sigma}^{(g)}$ then $S^{(g+1)}(x, y)$ is given by

$$
S^{(g+1)}(x, y)=S_{\kappa}^{(g)}(x, y)+\xi h(x) D^{\theta}\left(I+\xi Y D^{\theta}\right) \bar{h}(y)^{T},
$$

for infinite diagonal matrix $D^{\theta}(k, l)=\left[\begin{array}{cc}\theta_{g+1}^{-1} & 0 \\ 0 & -\theta_{g+1}\end{array}\right] \delta(k, l)$.

Proof. From (85) of Proposition 4.3 we find

$$
\begin{aligned}
& S^{(g+1)}(x, y)-S_{\kappa}^{(g)}(x, y)=\sum_{a=1,2} \frac{1}{2 \pi i} \oint_{\mathcal{C}_{a}\left(z_{a}\right)} S_{\kappa}^{(g)}\left(x, z_{a}\right) S^{(g+1)}\left(z_{a}, y\right) \\
= & \sum_{a=1,2} \sum_{k \geq 1} h_{a}(k, x) \frac{\rho^{-\frac{k_{a}}{2}+\frac{1}{4}}}{2 \pi i} \oint_{\mathcal{C}_{a}\left(z_{a}\right)} z_{a}^{k_{a}-1} S^{(g+1)}\left(z_{a}, y\right) d z_{\bar{a}}^{\frac{1}{2}} \\
= & \xi \sum_{a, k} h_{a}(k, x) D_{a a}^{\theta}(k, k) \frac{\rho^{\frac{k_{a}}{2}-\frac{1}{4}}}{2 \pi i} \oint_{\mathcal{C}_{\bar{a}}\left(z_{\bar{a}}\right)} z_{\bar{a}}^{-k_{a}} S^{(g+1)}\left(z_{\bar{a}}, y\right) d z_{\bar{a}}^{\frac{1}{2}}
\end{aligned}
$$

using respectively (94), (76) and (96). Applying (86) it follows that $S^{(g+1)}(x, y)-$ $S_{\kappa}^{(g)}(x, y)$ is given by

$$
\begin{aligned}
& \xi \sum_{a, k} h_{a}(k, x) D_{a a}^{\theta}(k, k) \frac{\rho^{\frac{k_{a}}{2}-\frac{1}{4}}}{2 \pi i} \oint_{\mathcal{C}_{\bar{a}}\left(z_{\bar{a}}\right)} z_{\bar{a}}^{-k_{a}} S_{\kappa}^{(g)}\left(z_{\bar{a}}, y\right) d z_{\bar{a}}^{\frac{1}{2}} \\
& -\xi \sum_{a, k} h_{a}(k, x) D_{a a}^{\theta}(k, k) \frac{\rho^{\frac{k_{a}}{2}-\frac{1}{4}}}{(2 \pi i)^{2}} \oint_{\mathcal{C}_{\bar{a}}\left(z_{\bar{a}}\right)} z_{\bar{a}}^{-k_{a} .} \\
& \sum_{b=1,2} \oint_{\mathcal{C}_{\bar{b}}\left(z_{\bar{b}}\right)} S^{(g+1)}\left(z_{\bar{a}}, w_{\bar{b}}\right) S_{\kappa}^{(g)}\left(w_{\bar{b}}, y\right) d z_{\bar{a}}^{\frac{1}{2}} \\
& =\xi h(x) D^{\theta} \bar{h}(y)^{T}-\xi \sum_{a, b, k, l} h_{a}(k, x) D_{a a}^{\theta}(k, k) . \\
& \frac{\rho^{\frac{1}{2}\left(k_{a}-l_{b}\right)}}{(2 \pi i)^{2}} \oint_{\mathcal{C}_{\bar{a}}\left(z_{\bar{a}}\right)} \oint_{\mathcal{C}_{\bar{b}}\left(w_{\bar{b}}\right)} z_{\bar{a}}^{-k_{a}} w_{\bar{b}}^{l_{b}-1} S^{(g+1)}\left(z_{\bar{a}}, w_{\bar{b}}\right) d z_{\bar{a}}^{\frac{1}{2}} d w_{\bar{b}}^{\frac{1}{2}} \bar{h}_{b}(l, y) \\
& =\xi h(x) D^{\theta} \bar{h}(y)^{T}-h(x) D^{\theta} Y D^{\theta} \bar{h}(y)^{T},
\end{aligned}
$$

on applying (95), (76) and (96). Hence the result follows. 
We next compute the explicit form of $Y$ in terms of the weighted moment matrix $G$ for $S_{\kappa}^{(g)}$. In particular it is convenient to define $T=\xi G D^{\theta}$. From Proposition 4.4 it follows on taking moments that

$$
Y=G+\xi G D^{\theta}\left(I+\xi Y D^{\theta}\right) G
$$

This can be solved recursively to obtain $Y=\sum_{n \geq 0} T^{n} G$. Following a similar argument to that given for Proposition 3.5 we then find

Proposition 4.5 $Y=(I-T)^{-1} G$ where $(I-T)^{-1}=\sum_{n \geq 0} T^{n}$ is convergent for $|\rho|<r_{1} r_{2}$.

This result together with Proposition 4.4 implies

Theorem $4.6 S^{(g+1)}(x, y)$ is given by

$$
S^{(g+1)}(x, y)=S_{\kappa}^{(g)}(x, y)+\xi h(x) D^{\theta}(I-T)^{-1} \bar{h}^{T}(y) .
$$

Finally, similarly to Theorem 3.9 we may define $\operatorname{det}(I-T)$ and find

Theorem $4.7 \operatorname{det}(I-T)$ is non-vanishing and holomorphic in $\rho$ for $|\rho|<$ $r_{1} r_{2}$.

\subsection{Self-Sewing a Sphere}

We consider the example of sewing the Riemann sphere $\Sigma^{(0)}=\mathbb{C} \cup\{\infty\}$ to itself to form a torus. Choose local coordinates $z_{2}=z \in \mathbb{C}$ in the neighborhood of the origin $p_{2}=0$, and $z_{1}=1 / z^{\prime}$ for $z^{\prime}$ in the neighborhood of the point at infinity $p_{1}=\infty$. Identify the annular regions $|q| r_{\bar{a}}^{-1} \leq\left|z_{a}\right| \leq r_{a}$ for a complex sewing parameter $\rho=q$ obeying $|q| \leq r_{1} r_{2}$, via the sewing relation

$$
z=q z^{\prime}
$$

These annular regions do not intersect on the sphere provided $r_{1} r_{2}<1$ so that $|q|<1$. Furthermore, the sewing relation implies $\log z=\log z^{\prime}+2 \pi i \tau+2 \pi i k$ for integer $k$ where $q=e^{2 \pi i \tau}$. This is the standard parameterization of the torus with periods $2 \pi i \tau$ and $2 \pi i$ and modular parameter $\tau \in \mathbb{H}_{1}$.

We now show that the results of the previous subsection allow us to recover the genus one Szegö kernel (23) from the genus zero one. For $x, y \in \mathbb{C}$ the genus zero prime form and Szegö kernel are given by

$$
\begin{aligned}
E^{(0)}(x, y) & =(x-y) d x^{-\frac{1}{2}} d y^{-\frac{1}{2}} \\
S^{(0)}(x, y) & =\frac{1}{x-y} d x^{\frac{1}{2}} d y^{\frac{1}{2}} .
\end{aligned}
$$


Let $\theta=\theta^{(1)}$ and $\phi=\phi^{(1)}=-e^{2 \pi i \kappa}$ denote the multipliers on the torus cycles. Then since $p_{1}=\infty$ and $p_{2}=0$ we find $U(x, y)=x / y$ so that (78) and (81) imply

$$
S_{\kappa}^{(0)}(x, y)=\frac{x^{\kappa} y^{-\kappa}}{x-y} d x^{\frac{1}{2}} d y^{\frac{1}{2}}+\frac{\theta}{1-\theta} \frac{d x^{\frac{1}{2}} d y^{\frac{1}{2}}}{x^{\frac{1}{2}} y^{\frac{1}{2}}} \delta_{\kappa,-\frac{1}{2}} .
$$

Computing moments one finds that for $\kappa \neq-\frac{1}{2}$ the half-differentials are

$$
\begin{aligned}
h_{1}(k, x) & =-\xi q^{\frac{1}{2}\left(k+\kappa-\frac{1}{2}\right)} x^{k+\kappa-1} d x^{\frac{1}{2}} \\
h_{2}(k, x) & =q^{\frac{1}{2}\left(k-\kappa-\frac{1}{2}\right)} x^{-k+\kappa} d x^{\frac{1}{2}} \\
\bar{h}_{1}(k, y) & =-q^{\frac{1}{2}\left(k+\kappa-\frac{1}{2}\right)} y^{-k-\kappa} d y^{\frac{1}{2}} \\
\bar{h}_{2}(k, y) & =\xi q^{\frac{1}{2}\left(k-\kappa-\frac{1}{2}\right)} y^{k-\kappa-1} d y^{\frac{1}{2}}
\end{aligned}
$$

for $x, y \in \widehat{\Sigma}^{(0)}$ and the moment matrix $T=\xi G D^{\theta}$ is diagonal with

$$
T_{a b}(k, l)=\theta^{a-\bar{a}} q^{k_{a}-\frac{1}{2}} \delta_{a b} \delta(k, l) .
$$

Altogether we find from Theorem 4.6 that for $\kappa \neq-\frac{1}{2}$ and $x, y \in \widehat{\Sigma}^{(0)}$

$$
\begin{aligned}
S^{(1)}(x, y)= & S_{\kappa}^{(0)}(x, y)+\xi h(x) D^{\theta}(I-T)^{-1} \bar{h}^{T}(y) \\
= & {\left[-\frac{\left(\frac{x}{y}\right)^{\kappa+\frac{1}{2}}}{1-\frac{x}{y}}-\sum_{k \geq 1} \frac{\theta^{-1} q^{k+\kappa-\frac{1}{2}}}{1-\theta^{-1} q^{k+\kappa-\frac{1}{2}}}\left(\frac{x}{y}\right)^{k+\kappa-\frac{1}{2}}\right.} \\
& \left.+\sum_{k \geq 1} \frac{\theta q^{k-\kappa-\frac{1}{2}}}{1-\theta q^{k-\kappa-\frac{1}{2}}}\left(\frac{y}{x}\right)^{k-\kappa-\frac{1}{2}}\right] \frac{d x^{\frac{1}{2}} d y^{\frac{1}{2}}}{x^{\frac{1}{2}} y^{\frac{1}{2}}} .
\end{aligned}
$$

Denoting $q_{u}=e^{u}$ for any $u$ we define $X, Y$ by $x=q_{X}, y=q_{Y}$ and let $Z=X-Y$. We also define $\lambda=\kappa+\frac{1}{2}$ with $0<\lambda<1$ and obtain

$$
\begin{aligned}
S^{(1)}(X, Y)= & {\left[-\frac{q_{Z}^{\lambda}}{1-q_{Z}}-\sum_{k \geq 0} \frac{\theta^{-1} q^{k+\lambda}}{1-\theta^{-1} q^{k+\kappa+\frac{1}{2}}} q_{Z}^{k+\lambda}\right.} \\
& \left.+\sum_{k \leq-1} \frac{\theta q^{-k-\lambda}}{1-\theta q^{-k-\lambda}} q_{Z}^{k+\lambda}\right] d X^{\frac{1}{2}} d Y^{\frac{1}{2}} \\
& =-\sum_{k \in \mathbb{Z}} \frac{q_{Z}^{k+\lambda}}{1-\theta^{-1} q^{k+\lambda}} d X^{\frac{1}{2}} d Y^{\frac{1}{2}}=P_{1}(Z, q) d X^{\frac{1}{2}} d Y^{\frac{1}{2}}
\end{aligned}
$$


from (24). A similar result also holds for $\kappa=-\frac{1}{2}$ for $\theta \neq 0$ i.e. $(\theta, \phi) \neq(0,0)$.

Lastly, we note that $(I-T)^{-1}$ is convergent for $|q|<1$ and that furthermore

$$
\operatorname{det}(I-T)=\prod_{k \geq 1}\left(1-\theta^{-1} q^{k+\kappa-\frac{1}{2}}\right)\left(1-\theta q^{k-\kappa-\frac{1}{2}}\right),
$$

is holomorphic for $|q|<1$ from Theorem 4.7. In vertex operator algebra theory, $\operatorname{det}(I-T)$ is related to the genus one partition function for a continuous orbfolding of a rank two free fermion system e.g. [MTZ]. Furthermore, the infinite product (101) is part of that arising in the Jacobi triple identity on applying the bosonic decomposition of this theory.

\subsection{Self-Sewing a Torus}

We next consider the example of self-sewing an oriented torus $\Sigma^{(1)}=\mathbb{C} / \Lambda$ for lattice $\Lambda=2 \pi i(\mathbb{Z} \tau \oplus \mathbb{Z})$ and $\tau \in \mathbb{H}_{1}$. This is discussed in detail in ref. [MT1. Define annuli $\mathcal{A}_{a}, a=1,2$ centered at $p_{1}=0$ and $p_{2}=w$ of $\Sigma^{(1)}$ with local coordinates $z_{1}=z$ and $z_{2}=z-w$ respectively. Take the outer radius of $\mathcal{A}_{a}$ to be $r_{a}<\frac{1}{2} D(q)$ for $D(q)=\min _{\lambda \in \Lambda, \lambda \neq 0}|\lambda|$ and the inner radius to be $|\rho| / r_{\bar{a}}$, with $|\rho| \leq r_{1} r_{2}$. Identifying the annuli via (66) we obtain a compact genus two Riemann surface $\Sigma^{(2)}$ parameterized by

$$
\mathcal{D}^{\rho}=\left\{(\tau, w, \rho) \in \mathbb{H}_{1} \times \mathbb{C} \times \mathbb{C}:|w-\lambda|>2|\rho|^{\frac{1}{2}}>0, \lambda \in \Lambda\right\} .
$$

For $x, y \in \Sigma^{(1)}$ the genus one prime form and Szegö kernel with multipliers $\theta_{1}=-e^{-2 \pi i \beta_{1}}$ and $\phi_{1}=-e^{2 \pi i \alpha_{1}}$ are given by (22) and (23). Let $\theta_{2}=-e^{-2 \pi i \beta_{2}}$ and $\phi_{2}=-e^{2 \pi i \alpha_{2}}=-e^{2 \pi i \kappa}$ denote the multipliers on $a_{2}, b_{2}$ cycles. Then, in this case

$$
U(x, y)=\frac{\vartheta_{1}(x-w, \tau) \vartheta_{1}(y, \tau)}{\vartheta_{1}(x, \tau) \vartheta_{1}(y-w, \tau)}
$$

and $z_{0, w}=\kappa w$ so that for $\kappa \neq-\frac{1}{2}$

$$
S_{\kappa}^{(1)}\left[\begin{array}{l}
\theta_{1} \\
\phi_{1}
\end{array}\right](x, y \mid \tau, w)=\left(\frac{\vartheta_{1}(x-w, \tau) \vartheta_{1}(y, \tau)}{\vartheta_{1}(x, \tau) \vartheta_{1}(y-w, \tau)}\right)^{\kappa} \frac{\vartheta\left[\begin{array}{l}
\alpha_{1} \\
\beta_{1}
\end{array}\right](x-y+\kappa w, \tau)}{\vartheta\left[\begin{array}{c}
\alpha_{1} \\
\beta_{1}
\end{array}\right](\kappa w, \tau) K(x-y, \tau)} d x^{\frac{1}{2}} d y^{\frac{1}{2}} \text {, }
$$

with a similar result for $\kappa=-\frac{1}{2}$. We take $\kappa \neq-\frac{1}{2}$ from now on. 
It is straightforward to see that

$$
S_{\kappa}^{(1)}\left[\begin{array}{l}
\theta_{1} \\
\phi_{1}
\end{array}\right](x, y \mid \tau, w)=S_{-\kappa}^{(1)}\left[\begin{array}{l}
\theta_{1} \\
\phi_{1}
\end{array}\right](x-w, y-w \mid \tau,-w) .
$$

Computing moments and using (93) and (103) the half-differentials (91), (92) for $x \in \widehat{\Sigma}^{(1)}$ and $\kappa \neq-\frac{1}{2}$ are given by

$$
\begin{aligned}
h_{1}\left[\begin{array}{l}
\theta_{1} \\
\phi_{1}
\end{array}\right](\kappa ; k, x \mid \tau, w, \rho)= & \frac{\rho^{\frac{1}{2}\left(k+\kappa-\frac{1}{2}\right)}}{2 \pi i}\left(\frac{\vartheta_{1}(x-w, \tau)}{\vartheta_{1}(x, \tau)}\right)^{\kappa} \frac{d x^{\frac{1}{2}}}{\vartheta\left[\begin{array}{l}
\alpha_{1} \\
\beta_{1}
\end{array}\right](\kappa w, \tau)} \\
& \oint_{\mathcal{C}_{1}(y)} y^{-k-\kappa}\left(\frac{\vartheta_{1}(y, \tau)}{\vartheta_{1}(y-w, \tau)}\right)^{\kappa} \frac{\vartheta_{\left[\begin{array}{l}
\alpha_{1} \\
\beta_{1}
\end{array}\right](x-y+\kappa w, \tau)}^{K(x-y, \tau)} d y,}{h_{2}\left[\begin{array}{l}
\theta_{1} \\
\phi_{1}
\end{array}\right](\kappa ; k, x \mid \tau, w, \rho)=} h_{1}\left[\begin{array}{l}
\theta_{1} \\
\phi_{1}
\end{array}\right](-\kappa ; k, x-w \mid \tau,-w, \rho), \\
\bar{h}_{1}\left[\begin{array}{l}
\theta_{1} \\
\phi_{1}
\end{array}\right](\kappa ; k, x \mid \tau, w, \rho)= & -h_{1}\left[\begin{array}{l}
\theta_{1}^{-1} \\
\phi_{1}^{-1}
\end{array}\right](\kappa ; k, x-w \mid \tau,-w, \rho), \\
\bar{h}_{2}\left[\begin{array}{l}
\theta_{1} \\
\phi_{1}
\end{array}\right](\kappa ; k, x \mid \tau, w, \rho)= & -h_{1}\left[\begin{array}{l}
\theta_{1}^{-1} \\
\phi_{1}^{-1}
\end{array}\right](-\kappa ; k, x \mid \tau, w, \rho) .
\end{aligned}
$$

Similarly, using (103), the moment matrix (89) is given by

$$
\begin{aligned}
G_{11}\left[\begin{array}{l}
\theta_{1} \\
\phi_{1}
\end{array}\right](\kappa ; k, l \mid \tau, w, \rho)= & \frac{\rho^{\kappa+\frac{1}{2}(k+l-1)}}{(2 \pi i)^{2}} \oint_{\mathcal{C}_{2}\left(x_{2}\right)} \oint_{\mathcal{C}_{1}\left(y_{1}\right)} x_{2}^{-k-\kappa} y_{1}{ }^{-l-\kappa} \\
& S_{\kappa}^{(1)}\left[\begin{array}{l}
\theta_{1} \\
\phi_{1}
\end{array}\right]\left(x_{2}, y_{1} \mid \tau, w\right) d x_{2}^{\frac{1}{2}} d y_{1}^{\frac{1}{2}} \\
& =G_{22}\left[\begin{array}{l}
\theta_{1} \\
\phi_{1}
\end{array}\right](-\kappa ; k, l \mid \tau,-w, \rho), \\
G_{21}\left[\begin{array}{l}
\theta_{1} \\
\phi_{1}
\end{array}\right](\kappa ; k, l \mid \tau, w, \rho)= & \frac{\rho^{\frac{1}{2}(k+l-1)}}{(2 \pi i)^{2}} \oint_{\mathcal{C}_{1}\left(x_{1}\right)} \oint_{\mathcal{C}_{1}\left(y_{1}\right)} x_{1}^{-k+\kappa} y_{1}^{-l-\kappa} \\
& S_{\kappa}^{(1)}\left[\begin{array}{l}
\theta_{1} \\
\phi_{1}
\end{array}\right]\left(x_{1}, y_{1} \mid \tau, w\right) d x_{1}^{\frac{1}{2}} d y_{1}^{\frac{1}{2}} \\
= & G_{12}\left[\begin{array}{l}
\theta_{1} \\
\phi_{1}
\end{array}\right](-\kappa ; k, l \mid \tau,-w, \rho) .
\end{aligned}
$$


The genus two Szego kernel is determined for $T=\xi G\left[\begin{array}{l}\theta_{1} \\ \phi_{1}\end{array}\right] D^{\theta_{2}}$ by (97)

$$
\begin{gathered}
S^{(2)}\left[\begin{array}{l}
\theta_{1} \\
\theta_{2}
\end{array}\right](x, y \mid \tau, w, \rho)= \\
S_{\kappa}^{(1)}\left[\begin{array}{l}
\theta_{1} \\
\phi_{1}
\end{array}\right](x, y \mid \tau, w)+\xi h\left[\begin{array}{l}
\theta_{1} \\
\phi_{1}
\end{array}\right](x) D^{\theta_{2}}(I-T)^{-1} \bar{h}^{T}\left[\begin{array}{l}
\theta_{1} \\
\phi_{1}
\end{array}\right](y) .
\end{gathered}
$$

\subsubsection{Modular Invariance}

We now consider the modular invariance of (106) under the action of a particular subgroup $L \subset S p(4, \mathbb{Z})$ and verify that $(20)$ holds. We define $L$ as follows [MT1]. Consider $\hat{H} \subset S p(4, \mathbb{Z})$ with elements

$$
\mu(a, b, c)=\left(\begin{array}{cccc}
1 & 0 & 0 & b \\
a & 1 & b & c \\
0 & 0 & 1 & -a \\
0 & 0 & 0 & 1
\end{array}\right)
$$

$\hat{H}$ is generated by $A=\mu(1,0,0), B=\mu(0,1,0)$ and $C=\mu(0,0,1)$ with relations $[A, B] C^{-2}=[A, C]=[B, C]=1$. We also define $\Gamma_{1} \subset S p(4, \mathbb{Z})$ where $\Gamma_{1} \cong S L(2, \mathbb{Z})$ with elements

$$
\gamma_{1}=\left(\begin{array}{cccc}
a_{1} & 0 & b_{1} & 0 \\
0 & 1 & 0 & 0 \\
c_{1} & 0 & d_{1} & 0 \\
0 & 0 & 0 & 1
\end{array}\right), \quad a_{1} d_{1}-b_{1} c_{1}=1
$$

Together these groups generate $L=\hat{H} \rtimes \Gamma_{1} \subset S p(4, \mathbb{Z})$ with center $Z(L)=$ $\langle C\rangle$ where $J=L / Z(L) \cong \mathbb{Z}^{2} \rtimes S L(2, \mathbb{Z})$ is the Jacobi group.

From Lemma 15 of [MT1] we find that $L$ acts on the domain $\mathcal{D}^{\rho}$ of (102) as follows:

$$
\begin{aligned}
\mu(a, b, c) \cdot(\tau, w, \rho) & =(\tau, w+2 \pi i a \tau+2 \pi i b, \rho) \\
\gamma_{1} \cdot(\tau, w, \rho) & =\left(\frac{a_{1} \tau+b_{1}}{c_{1} \tau+d_{1}}, \frac{w}{c_{1} \tau+d_{1}}, \frac{\rho}{\left(c_{1} \tau+d_{1}\right)^{2}}\right) .
\end{aligned}
$$

The kernel of the action is $Z(L)$, so that the effective action is that of $J$. However, this action is lifted to $L$ when considering the covering space $\widehat{\mathcal{D}}^{\rho}$ of $\mathcal{D}^{\rho}$ for which $\Omega_{g+1, g+1}^{(g+1)}$ of $(\underline{72})$ is single-valued (Theorems 10, 11 of [MT1]). In particular, one finds that $C$ acts as

$$
C .(\tau, w, \rho)=\left(\tau, w, e^{2 \pi i} \rho\right),
$$


which has a non-trivial action on $\widehat{\mathcal{D}}^{\rho}$.

Let us now consider the action of $L$ on $S^{(2)}\left[\begin{array}{l}\theta^{(2)} \\ \phi^{(2)}\end{array}\right](x, y \mid \tau, w, \rho)$. This is partly determined by the action of $J$ on $S_{\kappa}^{(1)}\left[\begin{array}{l}\theta_{1} \\ \phi_{1}\end{array}\right](x, y \mid w, \tau)$. For $\gamma_{1} \in \Gamma_{1}$ it is clear from (25) that

$$
S_{\kappa}^{(1)}\left[\begin{array}{c}
\theta_{1}^{a} \phi_{1}^{b} \\
\theta_{1}^{c} \phi_{1}^{d}
\end{array}\right]\left(\gamma_{1} x, \gamma_{1} y \mid \gamma_{1}(\tau, w)\right)=S_{\kappa}^{(1)}\left[\begin{array}{l}
\theta_{1} \\
\phi_{1}
\end{array}\right](x, y \mid \tau, w) .
$$

$h_{a}\left[\begin{array}{l}\theta_{1} \\ \phi_{1}\end{array}\right](\kappa ; k, x \mid \tau, w, \rho)$ and $G_{a b}\left[\begin{array}{l}\theta_{1} \\ \phi_{1}\end{array}\right](\kappa ; k, l \mid \tau, w, \rho)$ are similarly $\Gamma_{1}$ invariant so that $S^{(2)}\left[\begin{array}{l}\theta^{(2)} \\ \phi^{(2)}\end{array}\right](x, y \mid \tau, w, \rho)$ is $\Gamma_{1}$ invariant in a similar fashion to (65)).

Next we consider the action of the generators $A, B$ and $C$. We firstly note that (21) implies

$$
A\left[\begin{array}{l}
\theta_{1} \\
\theta_{2} \\
\phi_{1} \\
\phi_{2}
\end{array}\right]=\left[\begin{array}{c}
\theta_{1} \\
-\theta_{2} \theta_{1} \\
-\phi_{1} \phi_{2}^{-1} \\
\phi_{2}
\end{array}\right], \quad B\left[\begin{array}{l}
\theta_{1} \\
\theta_{2} \\
\phi_{1} \\
\phi_{2}
\end{array}\right]=\left[\begin{array}{c}
-\theta_{1} \phi_{2} \\
-\theta_{2} \phi_{1} \\
\phi_{1} \\
\phi_{2}
\end{array}\right], \quad C\left[\begin{array}{c}
\theta_{1} \\
\theta_{2} \\
\phi_{1} \\
\phi_{2}
\end{array}\right]=\left[\begin{array}{c}
\theta_{1} \\
-\theta_{2} \phi_{2} \\
\phi_{1} \\
\phi_{2}
\end{array}\right] .
$$

Using (8) and recalling that $\phi_{2}=-e^{2 \pi i \kappa}$ we find

$$
\begin{aligned}
S_{\kappa}^{(1)}\left[\begin{array}{l}
\theta_{1} \\
\phi_{1}
\end{array}\right](x, y \mid \tau, w) & =S_{\kappa}^{(1)}\left[\begin{array}{c}
\theta_{1} \\
-\phi_{1} \phi_{2}^{-1}
\end{array}\right](x, y \mid \tau, w+2 \pi i \tau) \\
& =S_{\kappa}^{(1)}\left[\begin{array}{c}
-\theta_{1} \phi_{2} \\
\phi_{1}
\end{array}\right](x, y \mid \tau, w+2 \pi i),
\end{aligned}
$$

where the multipliers comply with those of (112) for $A$ and $B$ respectively. Define infinite diagonal matrices

$$
E^{\alpha}(k, l)=\left[\begin{array}{cc}
1 & 0 \\
0 & -\alpha
\end{array}\right] \delta(k, l), \quad F^{\alpha}(k, l)=\left[\begin{array}{cc}
-\alpha^{-1} & 0 \\
0 & 1
\end{array}\right] \delta(k, l),
$$


for $\alpha \in U(1)$. Then (104), (113) and (114) imply

$$
\begin{aligned}
h\left[\begin{array}{l}
\theta_{1} \\
\phi_{1}
\end{array}\right](\kappa ; x \mid \tau, w, \rho) & =h\left[\begin{array}{c}
\theta_{1} \\
-\phi_{1} \phi_{2}^{-1}
\end{array}\right](\kappa ; x \mid \tau, w+2 \pi i \tau, \rho) E^{\theta_{1}} \\
& =h\left[\begin{array}{c}
-\theta_{1} \phi_{2} \\
\phi_{1}
\end{array}\right](\kappa ; x \mid \tau, w+2 \pi i, \rho) E^{\phi_{1}} \\
& =e^{-i \pi \kappa} h\left[\begin{array}{c}
\theta_{1} \\
\phi_{1}
\end{array}\right]\left(\kappa ; x \mid \tau, w, e^{2 \pi i} \rho\right) E^{\phi_{2}}, \\
\bar{h}\left[\begin{array}{c}
\theta_{1} \\
\phi_{1}
\end{array}\right](\kappa ; x \mid \tau, w, \rho) & =h\left[\begin{array}{c}
\theta_{1} \\
-\phi_{1} \phi_{2}^{-1}
\end{array}\right](\kappa ; x \mid \tau, w+2 \pi i \tau, \rho) F^{\theta_{1}} \\
& =h\left[\begin{array}{c}
-\theta_{1} \phi_{2} \\
\phi_{1}
\end{array}\right](\kappa ; x \mid \tau, w+2 \pi i, \rho) F^{\phi_{1}} \\
& =e^{i \pi \kappa} h\left[\begin{array}{c}
\theta_{1} \\
\phi_{1}
\end{array}\right]\left(\kappa ; x \mid \tau, w, e^{2 \pi i} \rho\right) F^{\phi_{2}} .
\end{aligned}
$$

Similarly, from (105) we find

$$
\begin{aligned}
G\left[\begin{array}{l}
\theta_{1} \\
\phi_{1}
\end{array}\right](\kappa \mid \tau, w, \rho) & =F^{\theta_{1}} G\left[\begin{array}{c}
\theta_{1} \\
-\phi_{1} \phi_{2}^{-1}
\end{array}\right](\kappa \mid \tau, w+2 \pi i \tau, \rho) E^{\theta_{1}} \\
& =F^{\phi_{1}} G\left[\begin{array}{c}
-\theta_{1} \phi_{2} \\
\phi_{1}
\end{array}\right](\kappa \mid \tau, w+2 \pi i, \rho) E^{\phi_{1}} \\
& =F^{\phi_{2}} G\left[\begin{array}{c}
-\theta_{1} \\
\phi_{1}
\end{array}\right]\left(\kappa \mid \tau, w, e^{2 \pi i} \rho\right) E^{\phi_{2}} .
\end{aligned}
$$

Noting that $E^{\alpha} D^{\theta_{2}} F^{\alpha}=D^{-\alpha \theta_{2}}$ for $\alpha=\theta_{1}, \phi_{1}$ and $\phi_{2}$ we may then easily confirm that $S^{(2)}\left[\begin{array}{l}\theta^{(2)} \\ \phi^{(2)}\end{array}\right](x, y \mid \tau, w, \rho)$ is invariant under the generators $A, B$ and $C$ respectively. Therefore $S^{(2)}\left[\begin{array}{l}\theta^{(2)} \\ \phi^{(2)}\end{array}\right](x, y \mid \tau, w, \rho)$ is modular invariant under $L$. Furthermore, since $\operatorname{det}\left(E^{\alpha} F^{\alpha}\right)=1$ it follows that $\operatorname{det}(I-T)$ is also $L$ invariant.

\section{References}

[DVFHLS] di Vecchia, P., Hornfeck, K., Frau, M., Lerda, A. and Sciuto, S.: N-string, g-loop vertex for the fermionic string, Phys.Lett. B211 (1988) 301-307. 
[DVPFHLS] di Vecchia, P., Pezzella, F., Frau, M., Hornfeck, K., Lerda, A. and Sciuto, S.: $N$-point $g$-loop vertex for a free fermionic theory with arbitrary spin, Nucl.Phys. B333 (1990) 635-700.

[F1] Fay, J.D.: Theta Functions on Riemann surfaces, Lecture Notes in Mathematics, Vol. 352. Springer-Verlag, (Berlin-New York, 1973).

[F2] Fay, J.D.: Kernel functions, analytic torsion, and moduli spaces, Mem.Amer.Math.Soc. 96 (1992) no. 464.

[FK] Farkas, H.M. and Kra, I.: Riemann Surfaces, Springer-Verlag (New York, 1980).

[FLM] Frenkel, I., Lepowsky, J. and Meurman, A.: Vertex Operator Algebras and the Monster, Academic Press (New York, 1988).

[FS] Freidan, D. and Shenker, S.: The analytic geometry of two dimensional conformal field theory, Nucl.Phys. B281 (1987) 509545 .

[HS] Hawley, N.S. and Schiffer, M.: Half-order differentials on Riemann surfaces, Acta Math. 115 (1966) 199-236.

[Ka] Kac, V.: Vertex Operator Algebras for Beginners, University Lecture Series, Vol. 10 AMS (Providence, 1998).

[MT1] Mason, G. and Tuite, M.P.: On genus two Riemann surfaces formed from sewn tori, Commun.Math.Phys. 270 (2007) 587634 .

[MT2] Mason, G. and Tuite, M.P.: Partition functions and chiral algebras, in Lie Algebras, Vertex Operator Algebras and their Applications (in honor of Jim Lepowsky and Robert Robert L. Wilson), Contemp.Math. 442 (2007) 401-410.

[MT3] Mason, G. and Tuite, M.P.: Free bosonic vertex operator algebras on genus two Riemann surfaces I, Commun.Math.Phys. 300 (2010) 673-713.

[MT4] Mason, G. and Tuite, M.P.: Free bosonic vertex operator algebras on genus two Riemann surfaces II, to appear. 
[MTZ] Mason, G., Tuite, M.P. and Zuevsky, A.: Torus n-point functions for $\mathbb{R}$-graded vertex operator superalgebras and continuous fermion orbifolds, Commun.Math.Phys. 283 (2008) 305342.

[Mu] Mumford, D.: Tata Lectures on Theta I and II, Birkhäuser, (Boston, 1983).

[R] Raina, A.K.: Fay's trisecant identity and conformal field theory, Commun.Math.Phys. 122 (1989) 625-641.

[RS] Raina, A.K. and Sen, S.: Grassmannians, multiplicative Ward identities and theta-function identities, Phys.Lett. B203 (1988) 256-262.

[Sc] Schiffer, M.: Half-order differentials on Riemann surfaces, SIAM J.Appl.Math. 14 (1966) 922-934.

[Sp] Springer, G.: Introduction to Riemann Surfaces, AddisonWesley (Reading, Mass. 1957).

[Sz] Szegö, G.: Über orthogonale Polynome, die zu einer gegebenen Kurve der komplexen Ebene gehören, Math.Z. 9 (1921) 218 270.

[T] Tuite, M.P: Genus two meromorphic conformal field theory, CRM Proceedings and Lecture Notes, 30 (2001) 231-251.

[TZ1] Tuite, M.P. and Zuevsky, A.: Genus two partition and correlation functions for fermionic vertex operator superalgebras I, arXiv:1007.5203, to appear in Commun.Math.Phys.

[TZ2] Tuite, M.P. and Zuevsky, A.: Genus two partition function for free fermionic vertex operator algebras II, to appear.

[Y] Yamada, A.: Precise variational formulas for abelian differentials, Kodai Math.J., 3 (1980) 114-143.

[Z] Zhu, Y.: Modular invariance of characters of vertex operator algebras, J.Amer.Math.Soc. 9 (1996) 237-302. 